Research Paper

\title{
A Novel Prognostic Predictor of Immune Micro- environment and Therapeutic Response in Kidney Renal Clear Cell Carcinoma based on Necroptosis-related Gene Signature
}

\author{
Wenwei Chen ${ }^{1,2}$, Wenfeng Lin ${ }^{3}$, Liang $\mathrm{Wu}^{4}$, Abai $\mathrm{Xu}^{1}{ }^{1}$, Chunxiao Liu ${ }^{1}$, Peng Huang ${ }^{1,3}{ }^{凶}$ \\ 1. Department of Urology, Zhujiang Hospital, Southern Medical University, Guangzhou, China. \\ 2. Department of Urology \& Department of Kidney Transplantation, The First Affiliated Hospital, Wenzhou Medical University, Wenzhou, China. \\ 3. Department of Urology, Okayama University Graduate School of Medicine, Dentistry and Pharmaceutical Sciences, Okayama, Japan. \\ 4. Department of Pathology, The First Affiliated Hospital, Wenzhou Medical University, Wenzhou, China \\ $\triangle$ Corresponding author: Peng Huang, Department of Urology, Okayama University Graduate School of Medicine, Dentistry and Pharmaceutical Sciences, \\ Okayama 700-8558, Japan. Tel.: + 81-86-235-7997; Fax +81-86-235-7884; Email: huangpeng509@gmail.com.
}

(c) The author(s). This is an open access article distributed under the terms of the Creative Commons Attribution License (https://creativecommons.org/licenses/by/4.0/). See http://ivyspring.com/terms for full terms and conditions.

Received: 2021.11.15; Accepted: 2022.01.11; Published: 2022.01.24

\begin{abstract}
Background: Necroptosis, a cell death of caspase-independence, plays a pivotal role in cancer biological regulation. Although necroptosis is closely associated with oncogenesis, cancer metastasis, and immunity, there remains a lack of studies determining the role of necroptosis-related genes (NRGs) in the highly immunogenic cancer type, kidney renal clear cell carcinoma (KIRC).

Methods: The information of clinicopathology and transcriptome was extracted from TCGA database. Following the division into the train and test cohorts, a three-NRGs (TLR3, FASLG, ZBP1) risk model was identified in train cohort by LASSO regression. The overall survival (OS) comparison was conducted between different risk groups through Kaplan-Meier analysis, which was further validated in test cohort. The Cox proportional hazards regression model was introduced to assess its impact of clinicopathological factors and risk score on survival. ESTIMATE and CIBERSORT algorithms were introduced to evaluate immune microenvironment, while enrichment analysis was conducted to explore the biological significance. Correlation analysis was applied for the correlation assessment between checkpoint gene expression and risk score, between gene expression and therapeutic response. Gene expressions from TCGA were verified by GEO datasets and immunohistochemistry (IHC) analysis.

Results: This NRGs-related signature predicted poorer OS in high-risk group, which was also verified in test cohort. Risk score could also independently predict survival outcome of KIRC. Significant changes were also found in immune microenvironment and checkpoint gene expressions between different risk groups, with immune functional enrichment in high-risk group. Interestingly, therapeutic response was correlated with the expressions of NRGs. The expressions of NRGs from TCGA were consistent with those from GEO datasets and IHC analysis.

Conclusion: The NRGs-related signature functions as a novel prognostic predictor of immune microenvironment and therapeutic response in KIRC.
\end{abstract}

Key words: prognosis, immune microenvironment, therapeutic response, kidney renal clear cell carcinoma, necroptosis, gene signature

\section{Introduction}

Kidney cancer incidence is rising globally, particularly in the younger population [1, 2]. In 2020, there are more than 431,000 new cases and 179,000 deaths from kidney cancer in the world [3]. Kidney renal clear cell carcinoma (KIRC), the most common histological subtype, is characterized by extensive tumor heterogeneity, distinct clinical courses, and potential specific treatment vulnerabilities [4]. Genetic alterations occur frequently in KIRC, such as somatic mutations of VHL, PBRM1, SETD2, BAP1, KDM5C, 
and PI3K-AKT-mTOR pathway genes [4, 5]. Although nephrectomy partially or radically showed good efficacy in treating localized KIRC, over $30 \%$ patients present with advanced-stage disease, and $25 \%$ of all patients ultimately experience disease relapse [6]. In recent years, the treatment scenario of advanced KIRC has evolved dramatically, with the emergence of targeted agents and immune checkpoint inhibitors (ICI). Indeed, real-life clinical practice faces the huge challenge of optimizing individualized therapeutic strategies. Biomarkers and prediction models can be applied for improving risk stratification and case selection for targeted therapy, immunotherapy, and combined therapeutics [4]. To date, no reliable predictive biomarkers have been identified for mirroring immune microenvironment and therapeutic response in KIRC.

Necroptosis is a caspase-independent necrotic cell death by genetical regulation, the main mediators of which include receptor interacting protein kinase 1 (RIPK1) and RIPK3, and mixed lineage kinase domain-like (MLKL) [7]. Accumulating evidence indicates that necroptosis plays a critical role in regulating oncogenesis and cancer progression, but it seems to be a double-edged sword [8]. For instance, the key mediator of necroptotic pathway, RIPK3 is downregulated in several cancer types, which associates with the increased tumor aggressiveness and chemoresistance [9-11]. This evidence suggests that necroptosis plays a positive role in anti-cancer progression. Conversely, the key necroptotic executioner, MLKL is upregulated in some cancers, correlating with highly invasive tumor behavior and immunosuppressive microenvironment [12, 13]. In addition, experimental studies indicate that cancer cells can induce the necroptosis of microvascular endothelial cells, thus promoting cancer cell extravasation and metastasis [14, 15]. Therefore, the function of necroptosis in cancer development is complex and context-dependent.

Interestingly, the spontaneous and mild necroptosis of tumor cells triggers pro-tumor immunity through releasing immunosuppressive molecules to modulate the tumor microenvironment including myeloid-derived suppressor cells (MDSC) and M2-like macrophages [16]. In contrast, the massive and acute necroptosis of tumor cells induced by chemotherapy or irradiation is often more immunogenetic, increasing the anti-tumor immunity through the activation of IFN- $\gamma$-expressing $\mathrm{T}$ cells [16]. Necroptosis has emerged as a promising cancer therapeutic target in combination with cancer immunotherapy. In the murine model, necroptosis induction of tumor cells in situ, can improve anti-tumor immunity when synergized with immune checkpoint blockade [17, 18]. Numerous key molecules in necroptotic pathways have been identified to be critical factors for cancer prognosis. The decreased MLKL expression is correlated with a reduced overall survival (OS) in ovarian carcinoma, gastric cancer, and colon cancer [19-21]. The upregulated RIPK1 expression associates with a poor prognosis in glioblastoma and breast cancer $[22,23]$.

However, it remained unclear whether necroptosis-related genes (NRGs) were associated with KIRC prognosis. This study was designed to construct a NRGs risk model in KIRC and determine its relationship with tumor immunity and therapeutic response. As presented in the flow process diagram (Figure 1), we developed a three-NRGs (TLR3, FASLG, ZBP1) signature which could predict OS, immune microenvironment, and therapeutic response in KIRC, followed by the verification of TCGA test cohort, GEO datasets, and clinical histological staining.

\section{Materials and methods}

\section{Data acquisition}

The collection of 12 necroptosis-related genes (NRGs) was conducted from the platform "http://www.gsea-msigdb.org/gsea/msigdb/search .jsp", including CASP8, CFLAR, FADD, FAS, FASLG, MLKL, RIPK1, RIPK3, TICAM1, TLR3, TNF, and ZBP1. TCGA database provided the information of clinicopathology and transcriptome with KIRC samples $(\mathrm{T})=539$ and normal samples $(\mathrm{N})=72$. GEO database provided two datasets including GSE40435 $(\mathrm{N}=101, \mathrm{~T}=101)$, and GSE53757 $(\mathrm{N}=72, \mathrm{~T}=72)$.

\section{Construction of a prognostic NRGs signature and its validation}

The expression data of 12 NRGs were firstly extracted from TCGA database, followed by the screening of differentially expressed genes (DEGs) between KIRC and normal tissues using $\mathrm{R}$ package "limma" with the filter conditions (fdrFilter $=0.05$, $\log$ FCfilter $=1$ ). After integrating the expression data of DEGs and survival data (more than 30 days), $\mathrm{R}$ package "caret" was applied to divide them into two cohorts (train cohort and test cohort). In train cohort, $\mathrm{R}$ package "glmnet" and the least absolute shrinkage and selection operator (LASSO) regression were applied for the NRGs risk signature identification. Following the risk score calculation using the formula: risk score $=\sum$ (gene expression $\times$ corresponding regression coefficient) [24], the subjects were classified into high-risk and low-risk groups based on the median score. $\mathrm{R}$ packages "survival" and "survminer" were introduced to evaluate overall 
survival (OS) based on Kaplan Meier (K-M) method. $\mathrm{R}$ package "timeROC" was applied for the generation of receiver operating characteristic (ROC) curve, while the area under the ROC curves (AUCs) of risk score, grade, and stage were used to evaluate the accuracy for predicting OS. Principal component analysis (PCA) was introduced for the exploration of group distribution using $\mathrm{R}$ package "ggplot2". Univariate and multivariate Cox proportional hazards regression models were introduced to evaluate the impact of risk score, and clinicopathological factors (age, gender, pathological grade, and clinical stage) on OS. The same analyses were conducted to validate the risk signature power in the test cohort. GSE40435 and GSE53757 datasets were further introduced to verify the expressions of FASLG, TLR3, and ZBP1.

\section{Immune microenvironment assessment}

The immune infiltration (immune score and stromal score) was assessed by the ESTIMATE algorithm [25], followed by analyzing the correlation of immune infiltration with risk score.

\section{Immune enrichment analysis}

Single sample gene set enrichment analysis (ssGSEA) was applied for the score calculation of immune cells and immune-related functions between different risk groups using R package "GSVA". As the annotated reference, "c5.all.v7.4.symbols.gmt" was introduced to GSEA software ( $\mathrm{v}$ 4.1.0) for the exploration of potential immunomodulatory functions.

\section{Drug response analysis}

From the CellMiner platform, we obtained the NCI-60 data, containing 60 types of cancer cell lines and the efficacy of FDA-approved drugs. The correlation of NRGs expressions with therapeutic response was assessed using Pearson correlation test.

\section{Immunohistochemistry (IHC) staining}

Eight pairs of paraffin-embedded KIRC and adjacent samples were collected in the First Affiliated Hospital of Wenzhou Medical University. Slices were baked at $65{ }^{\circ} \mathrm{C}$ for $2 \mathrm{~h}$, followed by dewaxing and antigen retrieval. After 10-min inactivation of endogenous enzymes by $3 \%$ hydrogen peroxide at room temperature, the slices underwent PBS rinsing 3 times for 3 min each. Following the blocking step by bovine serum albumin, the primary antibodies against FASLG, TLR3, and ZBP1 were applied to incubate the slices overnight at $4{ }^{\circ} \mathrm{C}$. Then washed them with PBS 3 times for $5 \mathrm{~min}$ each. After incubation with the secondary antibody at $37{ }^{\circ} \mathrm{C}$ for $30 \mathrm{~min}$, the slices

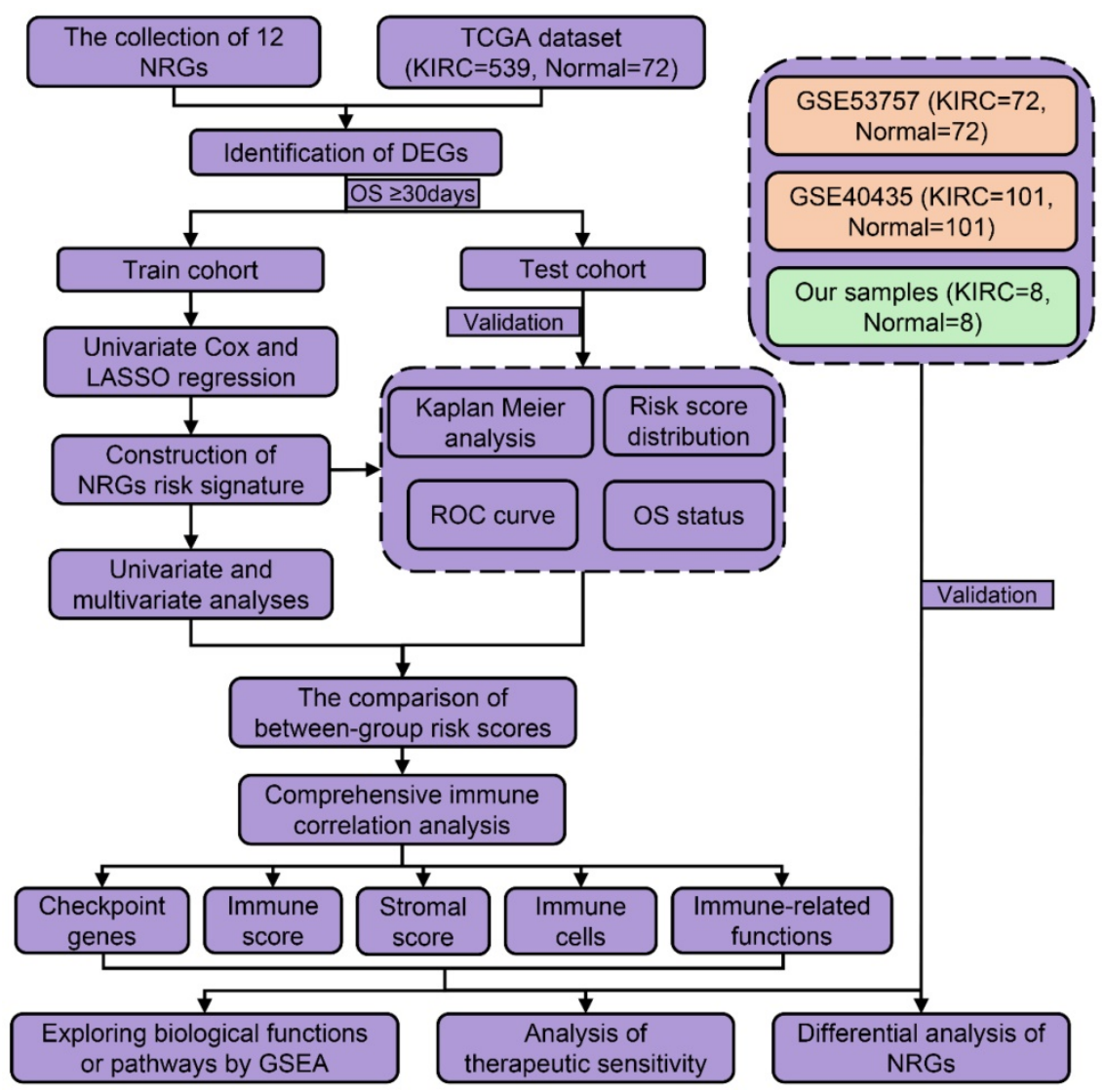

Figure 1. The flow process diagram of identifying NRGs risk model. 
underwent $\mathrm{DAB}$ color rendering for $5-10 \mathrm{~min}$, and hematoxylin redye for $3 \mathrm{~min}$. The slices were finally observed under microscope, followed by integrated optical density (IOD) measurement by Image Pro Plus 6.0 image software. The relative expressions of FASLG, TLR3, and ZBP1 were presented as average optical density (IOD/positive staining area).

\section{Statistical analysis}

$\mathrm{R}$ software (v 4.1.0), IBM SPSS software (v 22), and GraphPad Prism (v 8.3) were applied for all statistical analyses and diagram drawing. Chi-Square test and K-M method were used to compare the characteristics in Table 1 of train cohort, and test cohort. The survival comparison and independent OS predictors were analyzed using K-M method, univariate, and multivariate Cox proportional hazards regression model, respectively. Pearson or Spearman correlation test was applied to analyze the correlation concerning risk score. The two-way analysis of variance (ANOVA) in GraphPad Prism was conducted for the comparison of IHC quantitative results. $\mathrm{P}$ values less than 0.05 were identified as statistical differences.

Table 1. Characteristics of train cohort, test cohort, and entire cohort

\begin{tabular}{|c|c|c|c|c|}
\hline & $\begin{array}{l}\text { Train cohort, } \\
N=257\end{array}$ & $\begin{array}{l}\text { Test cohort, } \\
N=256\end{array}$ & $\begin{array}{l}\text { Entire cohort, } \\
N=513\end{array}$ & $\mathrm{P}^{*}$ \\
\hline & Number (\%) & Number (\%) & Number (\%) & \\
\hline Age & & & & 0.319 \\
\hline$\leq 65$ & 165 (64.2) & 175 (68.4) & $340(66.3)$ & \\
\hline$>65$ & $92(35.8)$ & 81 (31.6) & $173(33.7)$ & \\
\hline Gender & & & & 0.476 \\
\hline Female & $92(35.8)$ & $84(32.8)$ & $176(34.3)$ & \\
\hline Male & $165(64.2)$ & $172(67.2)$ & 337 (65.7) & \\
\hline Grade & & & & 0.778 \\
\hline $1-2$ & $115(44.7)$ & $116(45.3)$ & $231(45.0)$ & \\
\hline $3-4$ & 137 (53.3) & 137 (53.5) & 274 (53.4) & \\
\hline Unknown & $5(2.0)$ & $3(1.2)$ & $8(1.6)$ & \\
\hline Stage & & & & 0.312 \\
\hline I/II & $160(62.3)$ & $152(59.4)$ & $312(60.8)$ & \\
\hline III/IV & $97(37.7)$ & $102(39.8)$ & $199(38.8)$ & \\
\hline Unknown & $0(0.0)$ & $2(0.8)$ & $2(0.4)$ & \\
\hline Survival & & & & 0.935 \\
\hline Live & $172(66.9)$ & $172(67.2)$ & 344 (67.1) & \\
\hline Dead & 85 (33.1) & $84(32.8)$ & 169 (32.9) & \\
\hline
\end{tabular}

*Statistical analysis in age, gender, grade, stage, and survival between train cohort and test cohort.

\section{Results}

\section{Identification of differentially expressed NRGs with prognostic value}

We firstly divided 513 KIRC patients with survival time more than 30 days into train cohort $(\mathrm{N}=$ 257) and test cohort $(\mathrm{N}=256)$. Table 1 shows the characteristics of train cohort, test cohort, and entire cohort. From 12 NRGs mentioned above, we then screened 5 DEGs (FAS, FASLG, MLKL, TLR3, and
ZBP1) between KIRC and normal tissues. Among these DEGs, we further identified three prognostic genes in train cohort, including FASLG (hazard ratio, $\mathrm{HR}=1.314 ; 95 \%$ confidence interval, $95 \% \mathrm{CI}=$ 1.016-1.700; $\mathrm{P}=0.038)$, TLR3 $(\mathrm{HR}=0.744 ; 95 \% \mathrm{CI}=$ $0.623-0.889 ; \mathrm{P}=0.001)$, and $\mathrm{ZBP} 1(\mathrm{HR}=2.394 ; 95 \% \mathrm{CI}=$ 1.513-3.789; $\mathrm{P}<0.001)$ (Figure 2A).

\section{Construction and validation of the NRGs risk signature}

In train cohort, LASSO regression model analysis was conducted on the remaining prognostic NRGs (FASLG, TLR3, and ZBP1), which finally confirmed the least errors of three NRGs including FASLG, TLR3, and ZBP1 in the risk signature (Figure 2B-2C). The formula was listed as follows: risk score $=(0.115 \times$ FASLG expression $)+(-0.316 \times$ TLR3 expression $)+$ (0.658 $\times$ ZBP1 expression). K-M method was performed to determine the impact of risk signature on prognosis, finding that KIRC patients in low-risk group had a longer OS compared with those in high-risk group $(\mathrm{P}<0.001)$ (Figure 2D). The AUCs of risk score for predicting $1 / 3 / 5 / 7 / 10$-year OS were $0.707,0.635,0.667,0.715$, and 0.712 , respectively (Figure 2E). The AUCs of grade and stage for predicting 10-year OS were 0.594 and 0.675, respectively (Figure 2F). The same coefficients were applied for the risk score calculation in test cohort. Consistently, test cohort showed the similar result in survival outcome $(P<0.01)$ (Figure $2 \mathrm{G})$. The AUCs of risk score for predicting $1 / 3 / 5 / 7 / 10$-year OS were $0.669,0.626,0.670,0.662$, and 0.775 , respectively (Figure 2H). The AUCs of grade and stage for predicting 10-year OS were 0.685 and 0.668, respectively (Figure 2I). The risk score formula was also applied to predict OS between high- and low-risk groups in other subtypes of renal cell carcinoma (RCC), such as kidney renal papillary cell carcinoma (KIRP) and kidney chromophobe (KICH), which showed a poorer ability in predicting OS than KIRC subtype (Figure S1).

In train cohort, the distribution of ranked risk scores and individual survival status were shown in Figure 3A, 3B, indicating a longer survival time for KIRC patients in low-risk group. Moreover, the between-group distribution was discrete in PCA scatter plot (Figure 3C). The comparisons in Figure 3D suggested the upregulated expressions of FASLG and ZBP1, but the downregulated expression of TLR3 in high-risk group. Consistently, the results including risk score distribution, individual survival status, PCA scatter plot, and gene expression comparisons were all successfully validated in test cohort (Figure 3E-3H). 
A

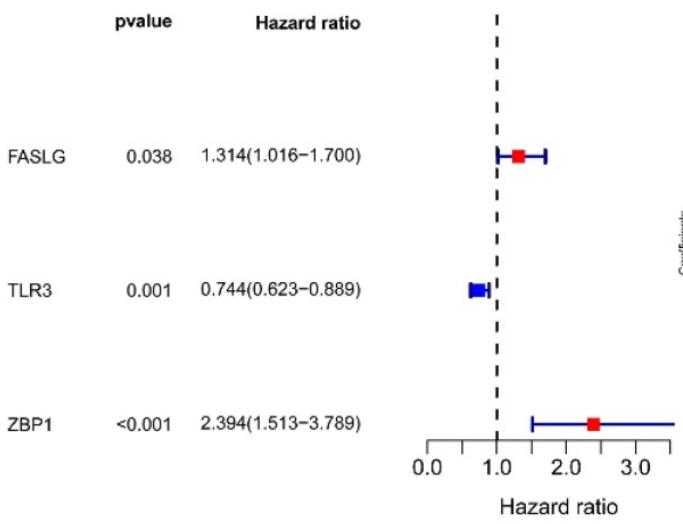

D

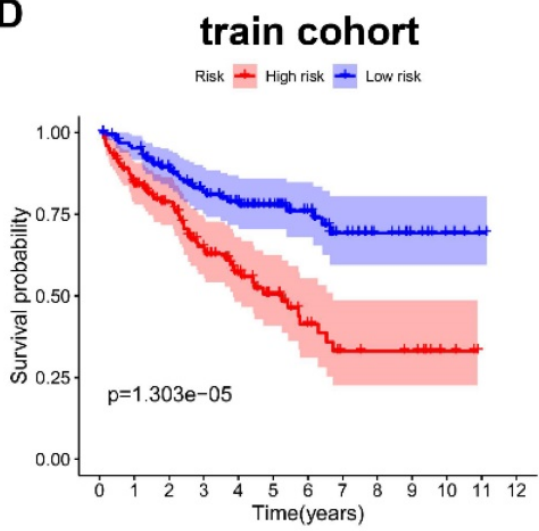

G

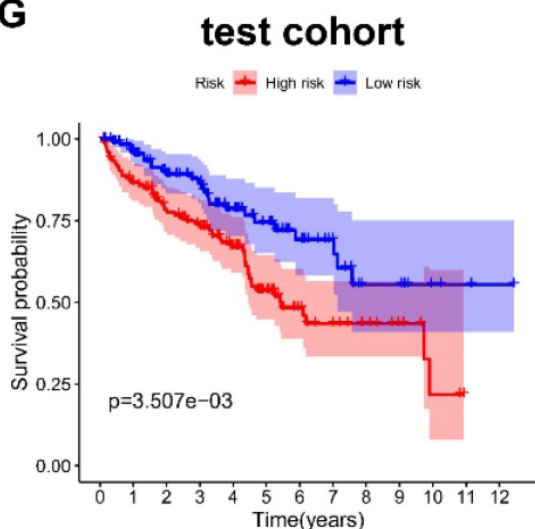

B

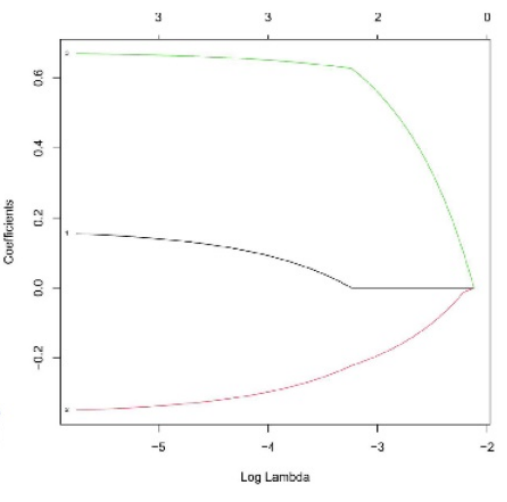

C

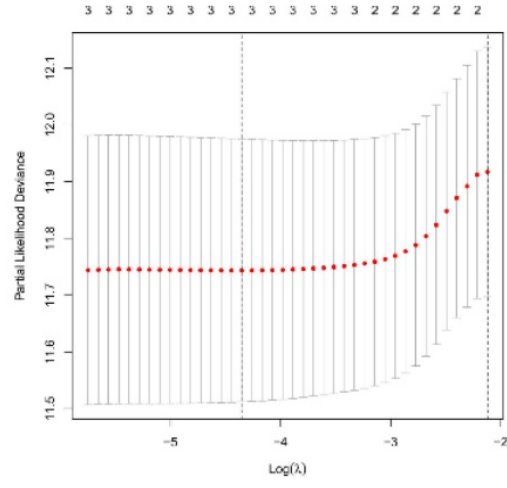

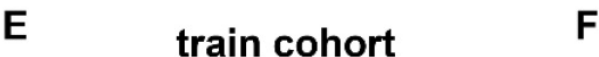

E train cohort

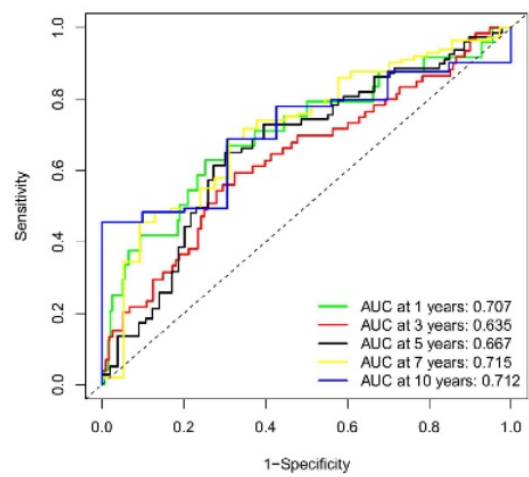

H test cohort

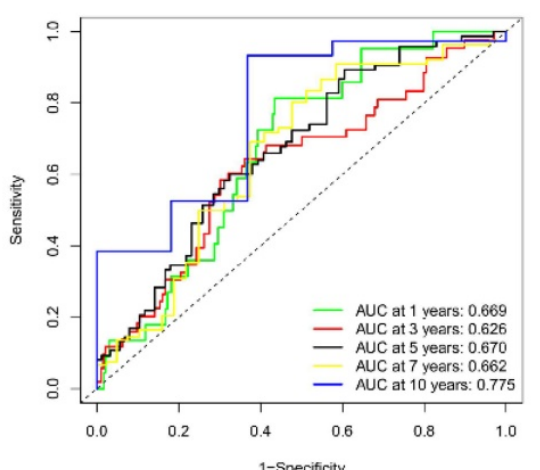

$\mathbf{F}$

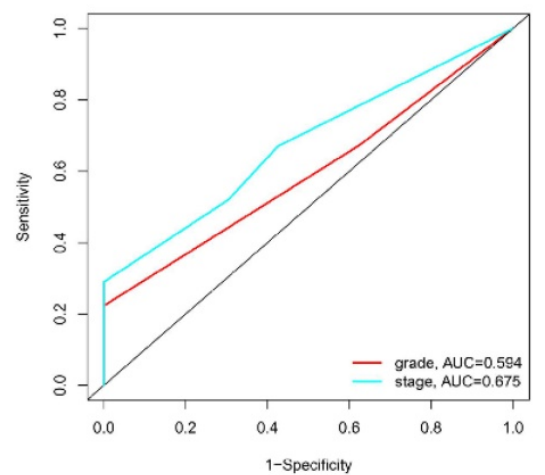

I

test cohort

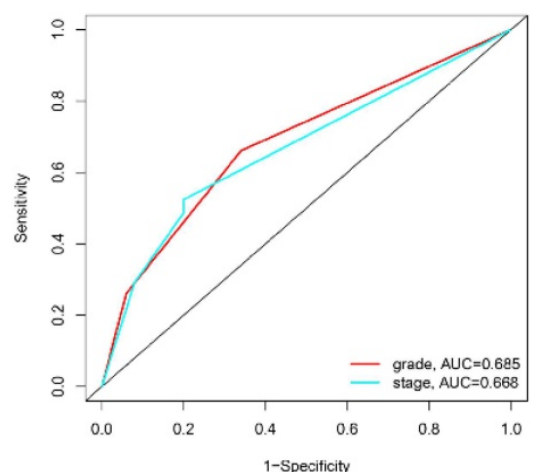

Figure 2. The NRGs risk model construction in KIRC. (A) The NRGs with prognostic values assessed by univariate Cox proportional hazards regression model in train cohort. (B-C) The selection of three-NRGs for risk model by LASSO analysis. K-M curves and time-dependent ROC curves for OS in (D-F) train cohort and (G-I) test cohort. Abbreviation: KIRC, kidney renal clear cell carcinoma; NRGs, necroptosis-related genes; LASSO, the least absolute shrinkage and selection operator; K-M, Kaplan-Meier; OS, overall survival.

\section{The risk score in three-NRGs signature independently predicts OS in KIRC}

Cox proportional hazards regression model was introduced for the screening of the OS independent predictors. In univariate analysis, the factors with significant difference were listed as follows: risk score $(\mathrm{HR}=3.106 ; 95 \% \mathrm{CI}=2.023-4.769 ; \mathrm{P}<0.001)$, clinical stage $(\mathrm{HR}=2.117 ; 95 \% \mathrm{CI}=1.734-2.584 ; \mathrm{P}<0.001)$, and pathological grade $(\mathrm{HR}=2.265 ; 95 \% \mathrm{CI}=1.697-3.021$; $\mathrm{P}<0.001$ ) (Figure 4A). Univariate regression analysis indicated no statistical difference in gender which was not included in multivariate Cox proportional hazards regression model. Therefore, multivariate Cox proportional hazards regression model was further applied to analyze these significant factors, finding that the independent predictors for OS included risk score $(\mathrm{HR}=2.717 ; 95 \% \mathrm{CI}=1.706-4.329$; $\mathrm{P}<0.001)$, clinical stage $(\mathrm{HR}=1.947 ; 95 \% \mathrm{CI}=$ 1.542-2.458; $\mathrm{P}<0.001$ ) (Figure 4B). 
A

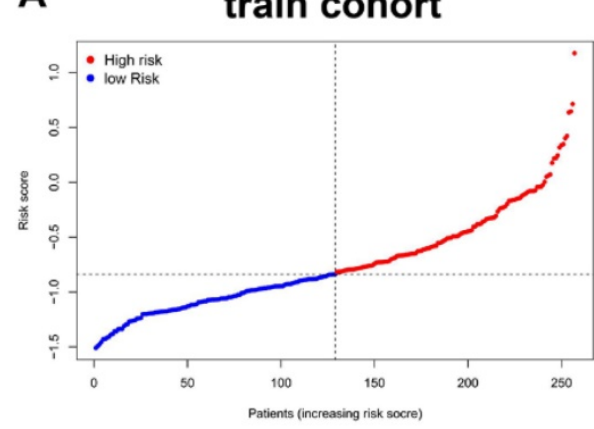

D

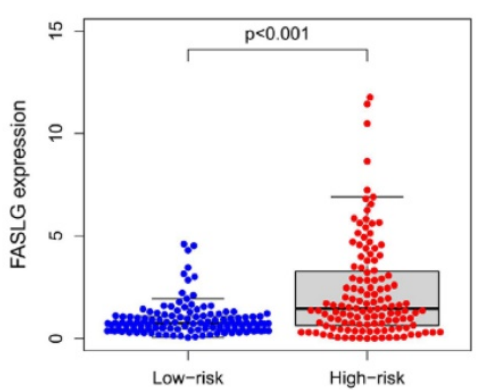

E

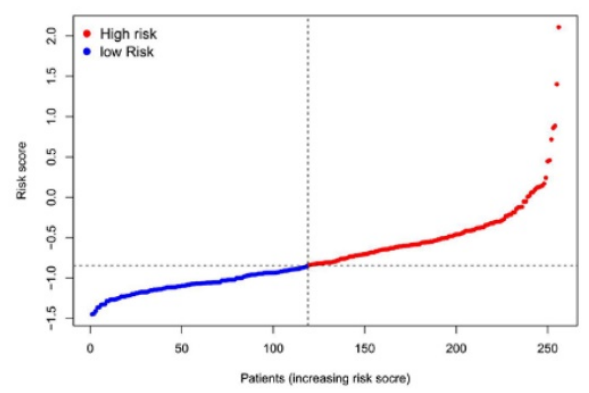

H

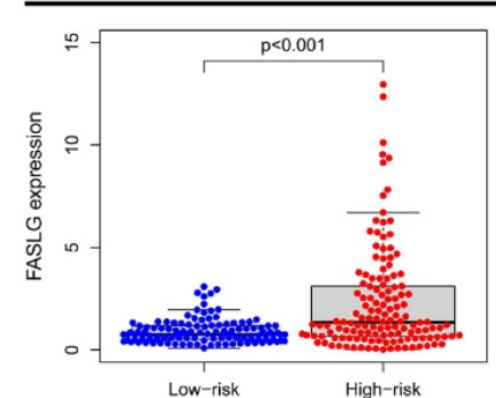

B

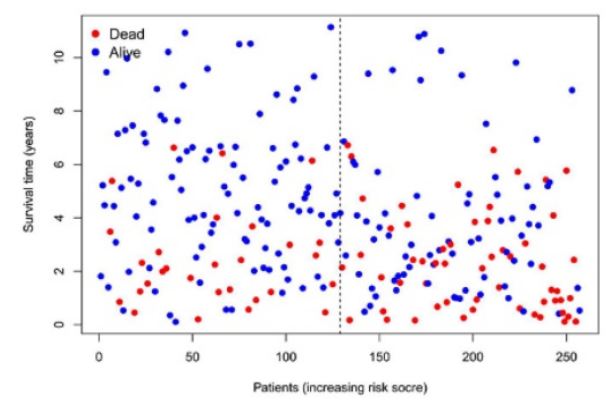

C train cohort

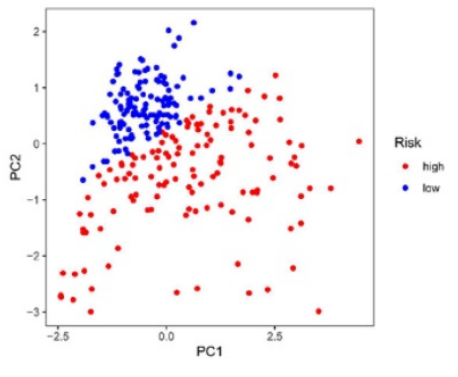

train cohort

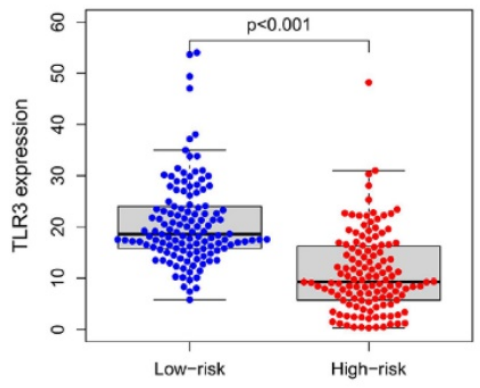

F

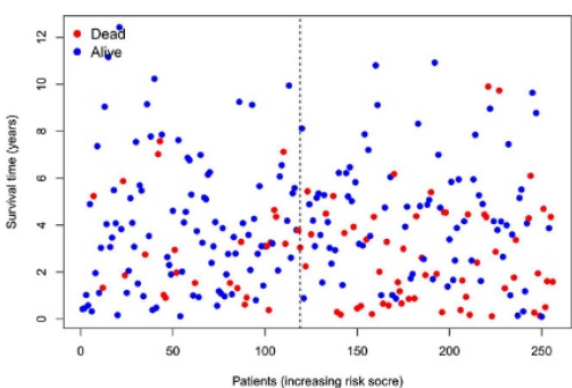

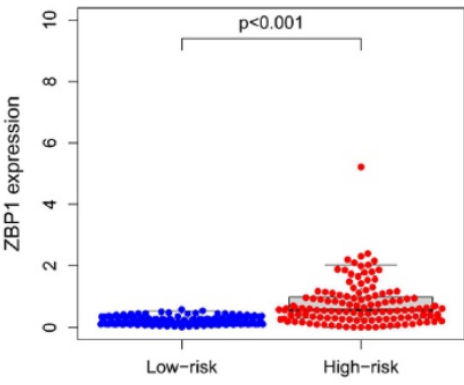

G test cohort

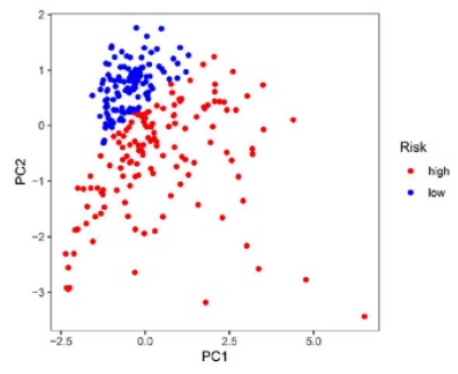

test cohort
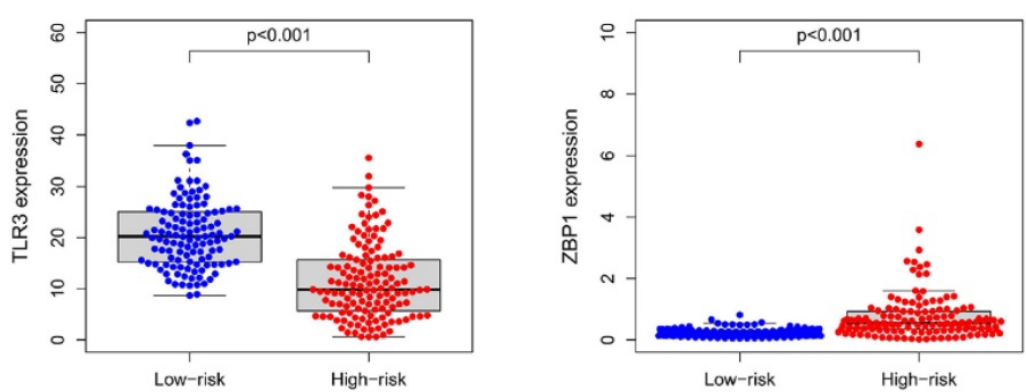

Figure 3. Validation of this NRGs-related risk model. (A) The risk score distribution and (B) OS status in train cohort. (C) PCA plot and (D) the comparison of FASLG, TLR3, and ZBPI expressions between high- and low-risk groups in train cohort. (E) The risk score distribution and (F) OS status in test cohort. (G) PCA plot and (H) the comparison of FASLG, TLR3, and ZBPI expressions between high- and low-risk groups in test cohort.

Differential risk score was further compared between groups based on clinicopathological factors. G3-4 and stage III-IV groups had higher risk scores than G1-2 $(\mathrm{P}<0.01)$ and stage I-II $(\mathrm{P}<0.01)$, but no statistical difference was found in risk scores between groups of age and gender in train cohort (Figure
4C-4F), which was successfully validated in test cohort (Figure 4G-4J).

\section{The NRGs signature closely associates with tumor immunity}

The ESTIMATE algorithm was introduced to assess immune infiltration, showing a positive 
correlation of risk score with immune score $(\mathrm{P}<0.001$; Figure 5A), but no statistical difference was found between risk score and stromal score (Figure 5B). The score comparisons were performed in 16 immune cell types and 13 kinds of immune-related functions between different risk groups. Among these immune cells, 9 types including B cells, CD8 positive T cells, Macrophages, pDCs, T helper cells, Tfh, Th1 cells, Th2 cells, and TIL, had higher scores in high-risk group than in low-risk group (Figure 5C). Among these immune-related functions, 12 kinds including Parainflammation, MHC class I, inflammation promoting, APC co-inhibition or co-stimulation, check point, Cytolytic activity, CCR, T cells co-inhibition or co-stimulation, HLA, and Type I IFN Response, had higher scores in high-risk group than in low-risk group, except Type II IFN Response (Figure 5D). We further enriched the immunomodulatory functions, including regulation of monocyte differentiation, regulation of $\mathrm{T}$ cell activation, $\mathrm{T}$ helper 1 type immune response, $\mathrm{T}$ helper cell lineage commitment, and abnormal proportion of CD8 positive $T$ cells (Figure 5E). In addition, the expressions of immune checkpoint genes (CTLA-4, LAG-3, PD-1, and SIGLEC15) were positively correlated with risk scores, and were significantly higher in high-risk group (Figure 6A-6H).
A

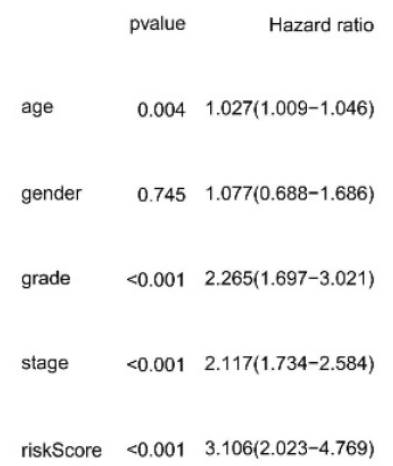

C

train cohort-grade

grade 审 91-2 审 63-4

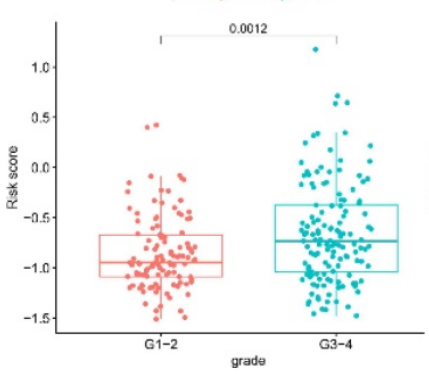

G test cohort-grade

grade 由i 61-2 由9 63-4

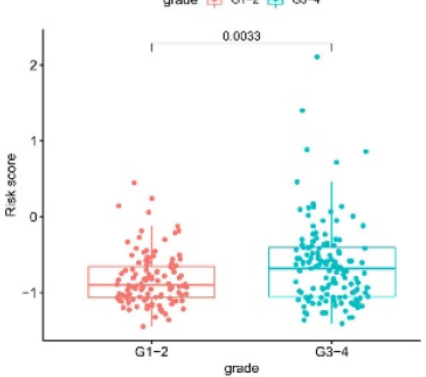

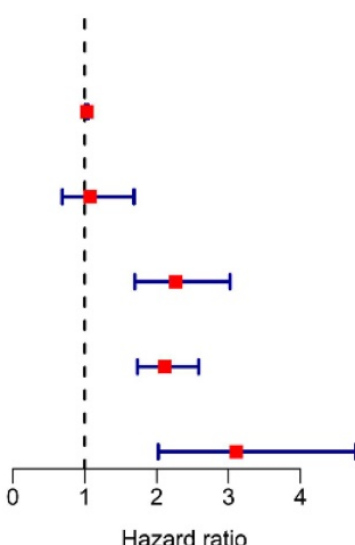

D train cohort-stage

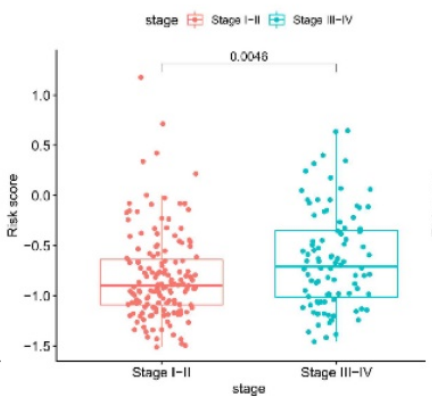

H test cohort-stage
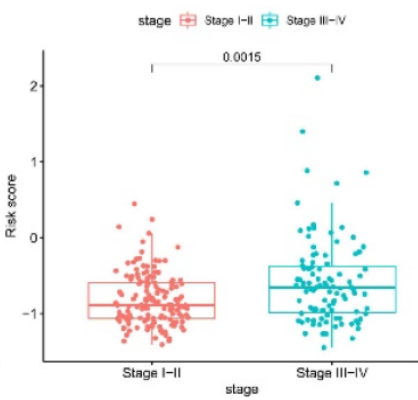

B

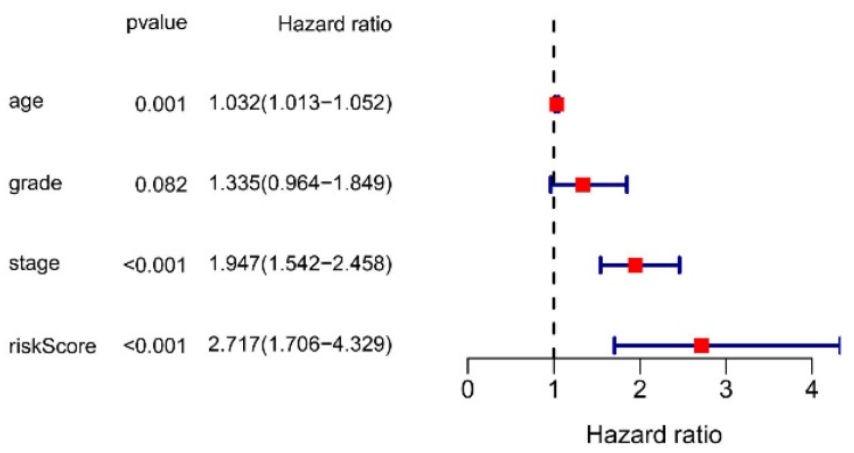

E train cohort-age

F

train cohort-gender
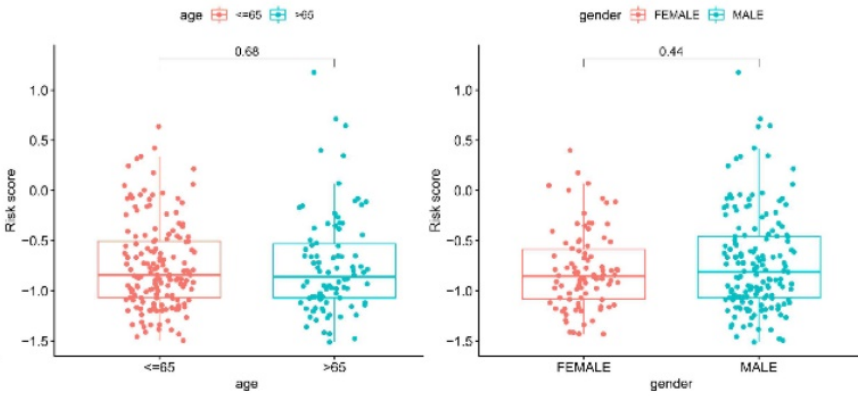

I

test cohort-age
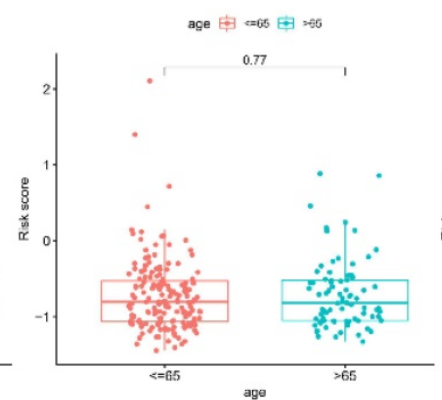

J

test cohort-gender

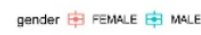

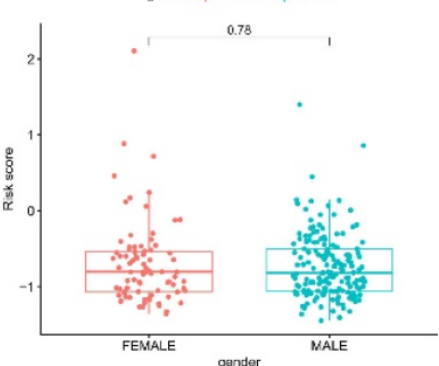

Figure 4. Prognostic values of clinicopathological factors and risk score. (A-B) Screening the independent predictors for OS in KIRC by univariate and multivariate Cox proportional hazards regression model. Since univariate regression analysis indicated no statistical difference in gender, it was not included in multivariate Cox proportional hazards regression model. The comparison of risk score between groups based on (C) pathological grade, (D) clinical stage, (E) age, and (F) gender in train cohort. The comparison of risk score between groups based on $(\mathbf{G})$ pathological grade, $(\mathbf{H})$ clinical stage, (I) age, and $(\mathbf{J})$ gender in test cohort. 
A

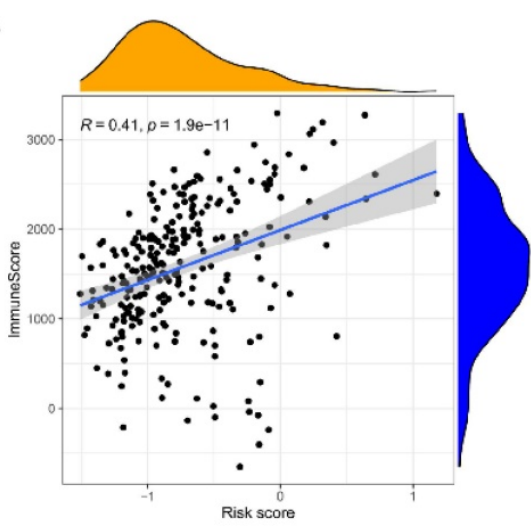

C

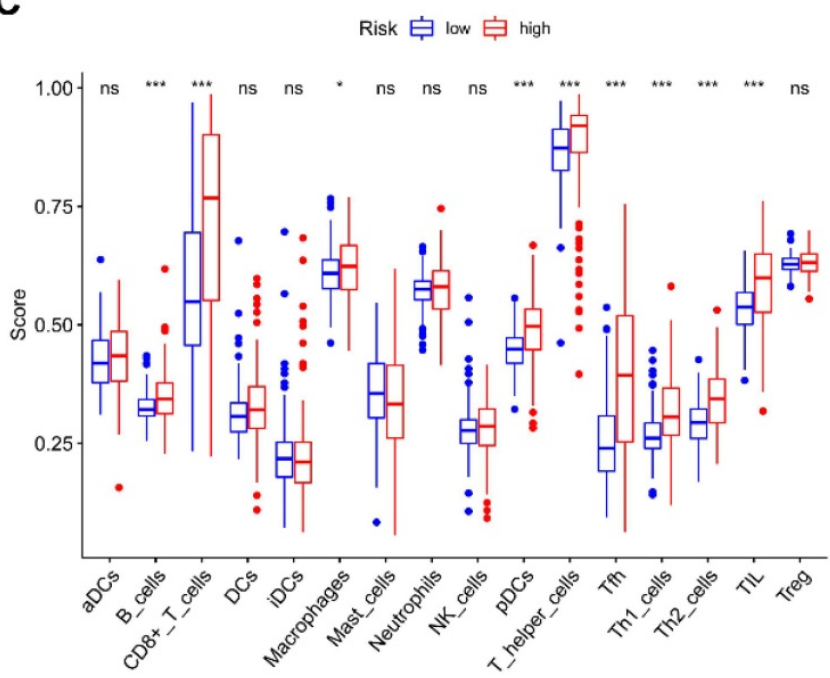

E
B

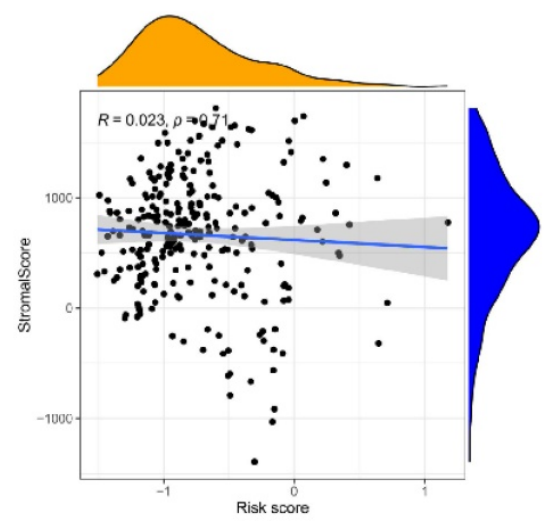

D

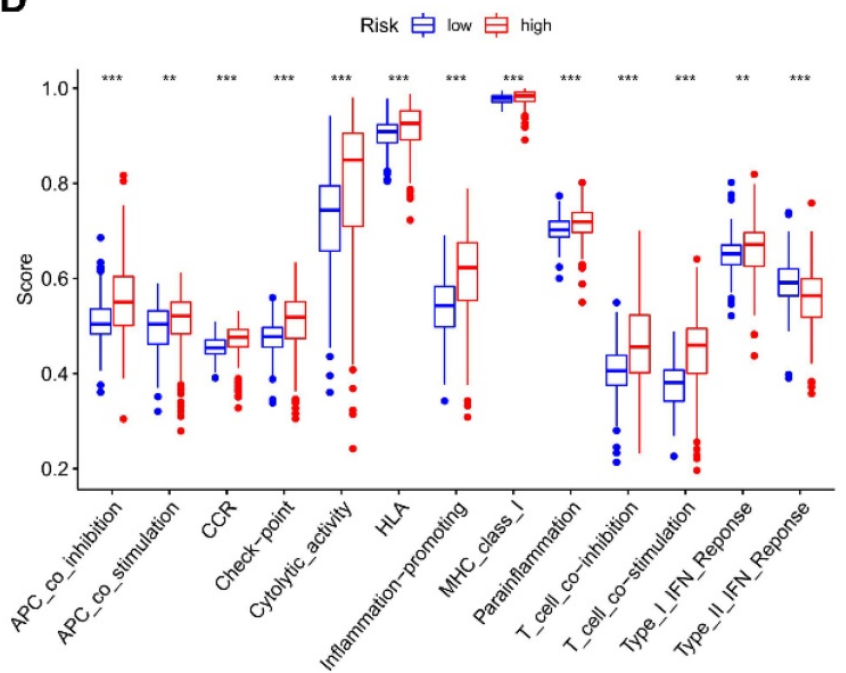

- GOBP_REGULATION_OF_MONOCYTE_DIFFERENTIATION

- GOBP_REGULATION_OF_T_CELL_ACTIVATION

- GOBP_T_HELPER_1_TYPE_IMMUUNE_RESPONSE

- GOBP_T_HELPER_CELL_LINEAGE_COMMITMENT

- HP_ABNORMAL_PROPORTION_OF_CD8 POSITIVE T CELLIS

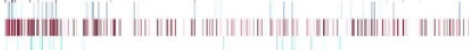

Figure 5. Correlation of NRGs-related signature with immune microenvironment. The association between risk score and (A) immune score, or (B) stromal score. Boxplots comparing the scores of (C) immune cells and (D) immune-related functions between different risk groups. (E) Immune functions enriched in high-risk group.

\section{The expressions of NRGs associates with therapeutic response}

To explore the potential clinical significance of NRGs signature, we integrated the data concerning cancer cell expressions and the efficacy of FDA-approved drugs. Cancer cells with higher FASLG expression were more sensitive to LEE-011, oxaliplatin, and palbociclib (Figure 7A). Cancer cells with higher TLR3 expression were more sensitive to JNJ-42756493 and IPI-145 (Figure 7B), but they were correlated with increased drug resistance to tyrothricin. Cancer cells with higher ZBP1 expressions were more sensitive to the following drugs including LDK-378, alectinib, and brigatinib (Figure 7C). 
A

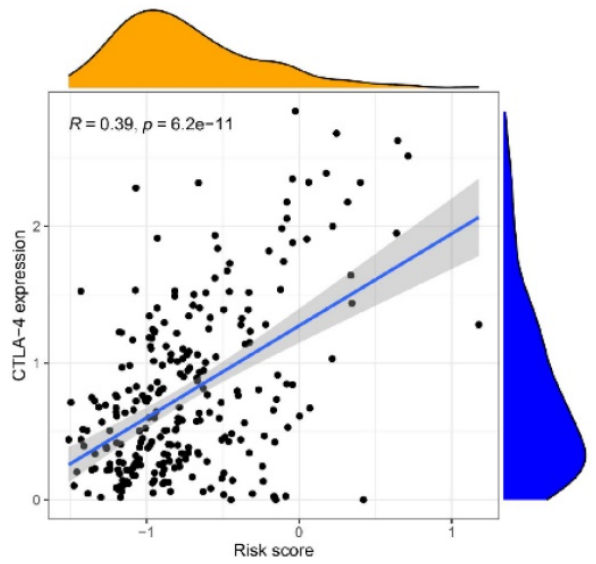

C

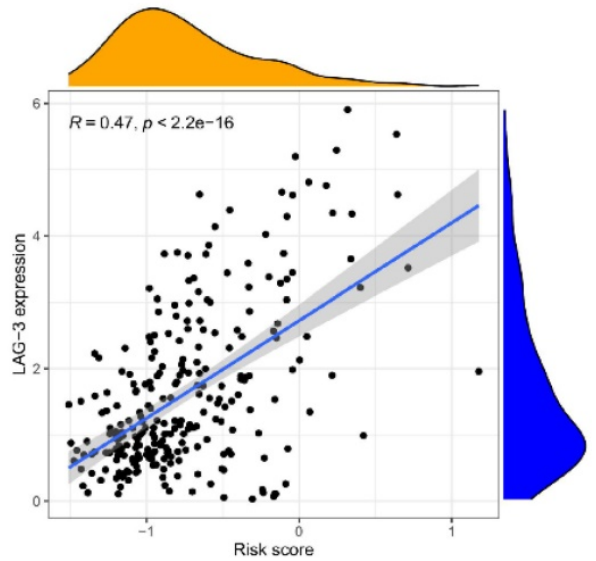

E

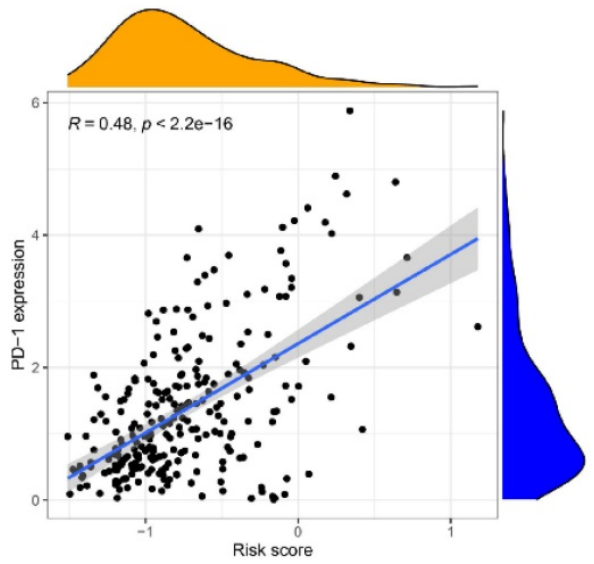

G

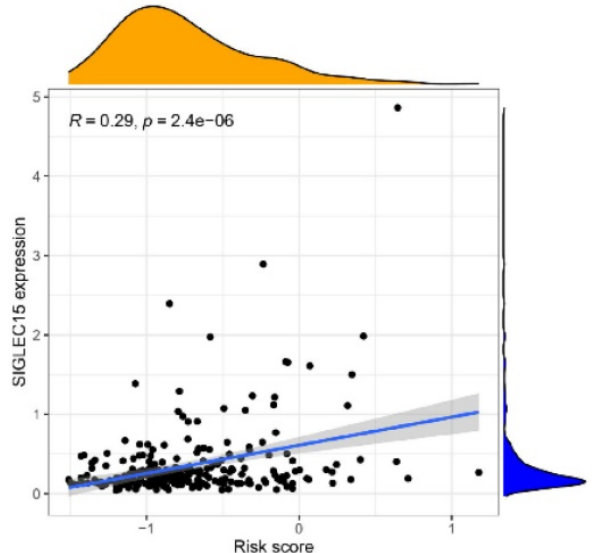

B

审 Low-risk 审 High-risk

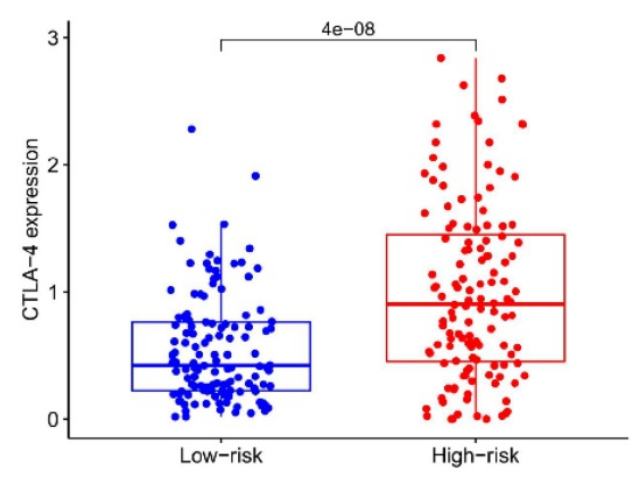

D

审 Low-risk 审 High-risk

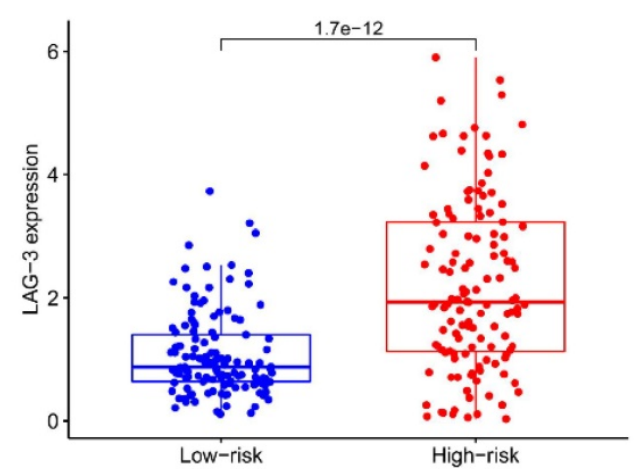

F

审 Low-risk 审 High-risk

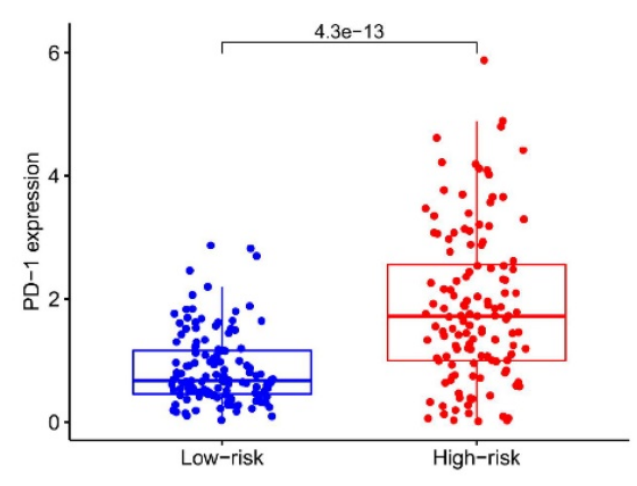

H

审 Low-risk

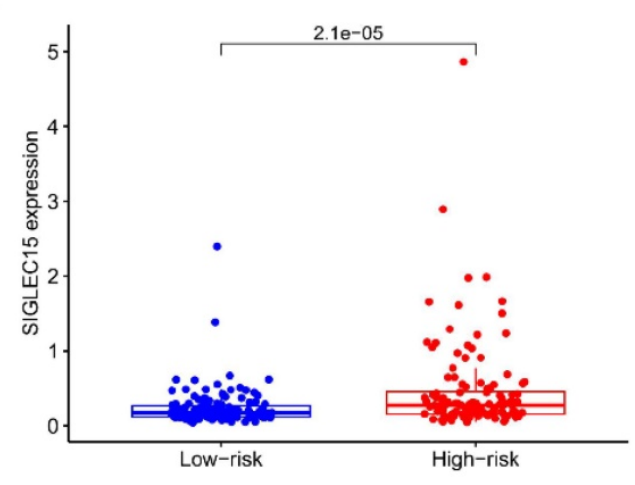

Figure 6. Correlation of NRGs-related signature with checkpoint gene expressions. The association of risk score with the expression levels of (A-B) CTLA-4, (C-D) LAG-3, (E-F) PD-1, and (G-H) SIGLEC15. 
A

FASLG

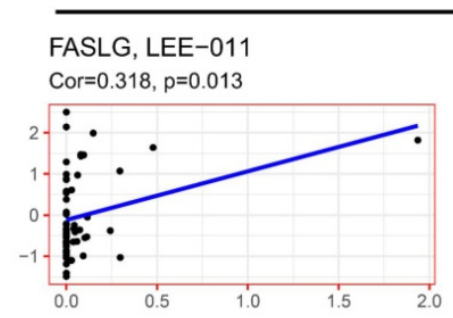

B
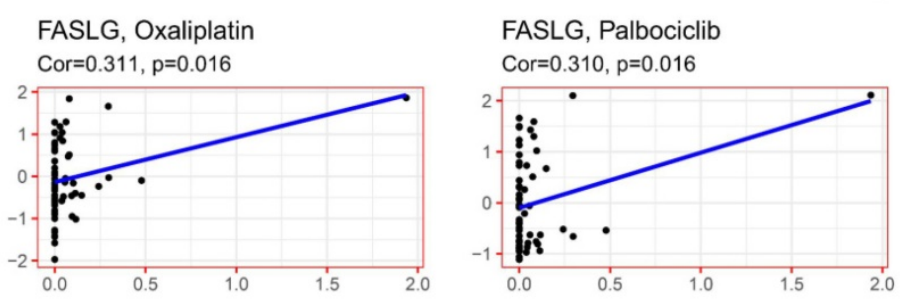

TLR3
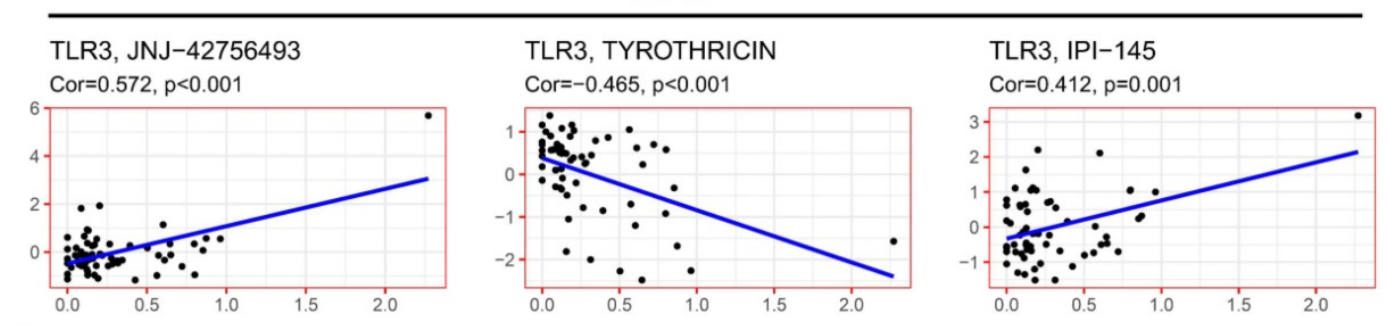

C

\section{ZBP1}
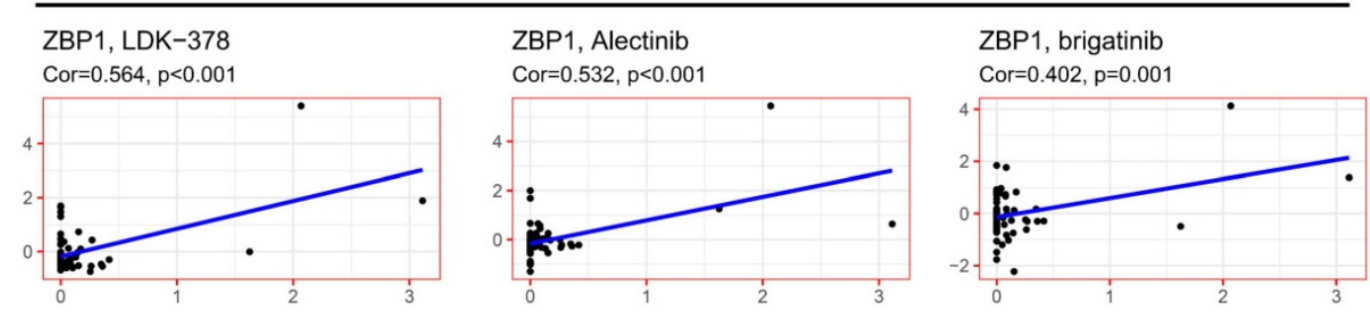

Figure 7. Correlation of NRGs expressions with therapeutic response. The association between drug sensitivity and the expressions of (A) FASLG, (B) TLR3, and (C) ZBPI.

\section{Differential expressions of NRGs between KIRC and normal samples}

Through TCGA database, we found the significant upregulation of FASLG, TLR3, and ZBP1 expressions both in train cohort and test cohort $(\mathrm{P}<$ 0.001; Figure 8A-8B). Consistently, in GSE53757 and GSE40435 datasets, the expressions of the three genes were significantly higher in KIRC tissues than in normal ones $(\mathrm{P}<0.001$; Figure 8C-8D). At the histological level, we further used IHC staining to validate the differential expressions of FASLG, TLR3, and ZBP1 in KIRC samples (Figure 8E-8H).

\section{The construction of a NRGs nomogram for predicting survival}

To promote the clinical value of this novel risk model, a nomogram including risk score and clinicopathological features was constructed to predict 1/3/5/7/10-year OS (Figure 9A). The calibration plots for predicting $1 / 3 / 5 / 7 / 10$-year OS based on the NRGs nomogram exhibited a favorable agreement of actual probability with the predicted probability (Figure 9B). To highlight the role of risk score in this nomogram, we constructed a second nomogram including only clinicopathological features, showing the inferior prediction effect especially in predicting 7- and 10-year OS (Figure S2).

\section{Discussion}

Clear cell renal cell carcinoma (ccRCC), also known as KIRC, is featured by angiogenic, inflammatory, and highly immunogenic microenvironment, showing different sensitivity to anti-angiogenic drugs and immunotherapy. The exploration of risk prediction model remains a big challenge to the selection of precision medicine for KIRC patients. New evidence suggests that RCC cells with high grade exhibit high levels of RIPK1 and RIPK3, which are more susceptible to necroptosis triggered by TNF receptor 1 [26]. Necroptotic pathway is involved in tumor necrosis [27], contributing to about half of the necrosis in head and neck squamous cell carcinoma [28]. In KIRC, necrosis is a common pathological phenomenon correlating with invasive phenotypes and poor prognosis [29]. In addition, necroptosis is correlated with microvascular invasion which has potential prognostic value in RCC [30]. These evidence leads to the speculation that tumor spontaneous necroptosis is associated with adverse clinical outcome of KIRC. As a cancer type of high immunogenicity, KIRC microenvironment 
exhibits a high-level T cell infiltration [31]. Within the spectrum of immunogenic cell death and drug resistance, necroptosis targeting has emerged as a potent anti-cancer therapeutic strategy [32, 33].
Therefore, this study explored the potential impact of NRGs signature on KIRC microenvironment and its corresponding therapeutic response.

A

train cohort

B
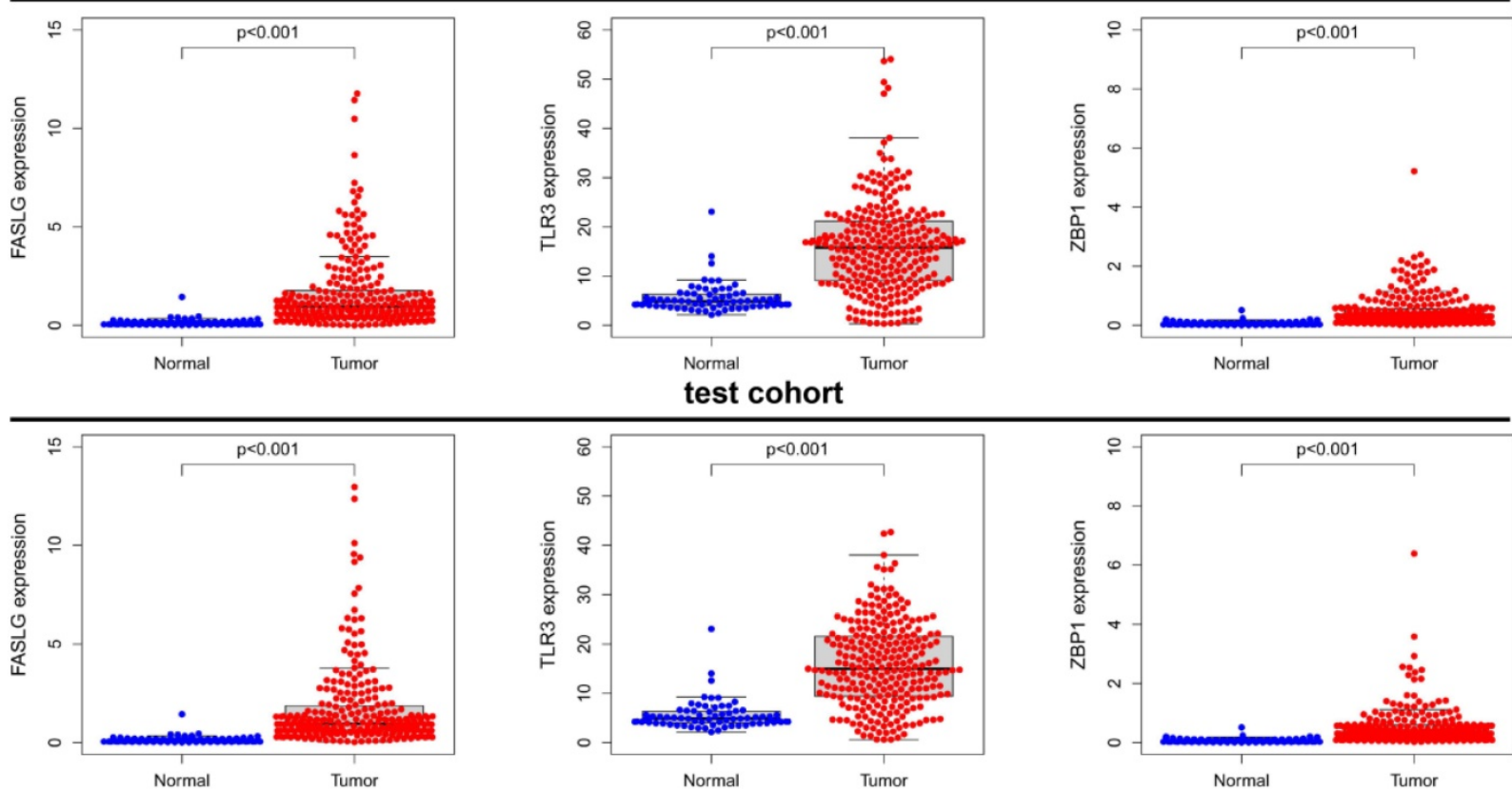

test cohort

C

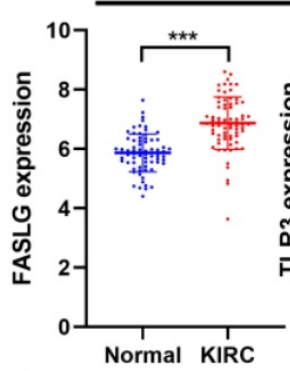

E

GSE53757

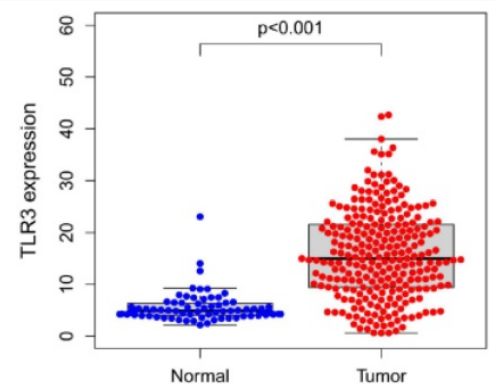

D

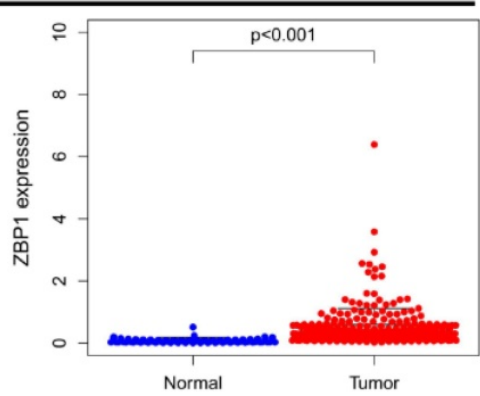

GSE40435

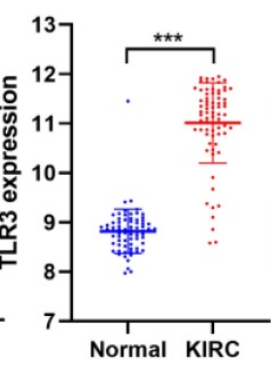

FASLG

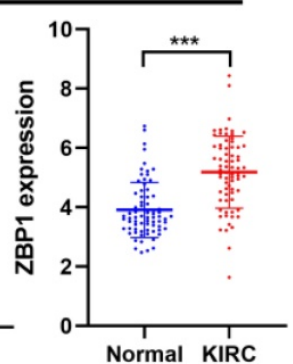

Normal KIRC

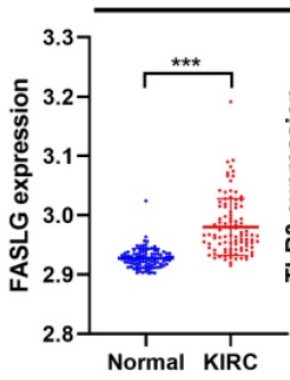

$\mathbf{F}$

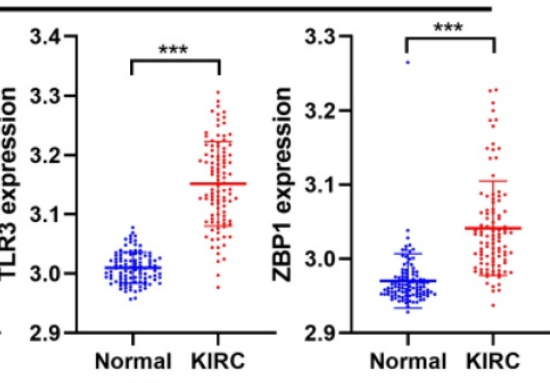

TLR3
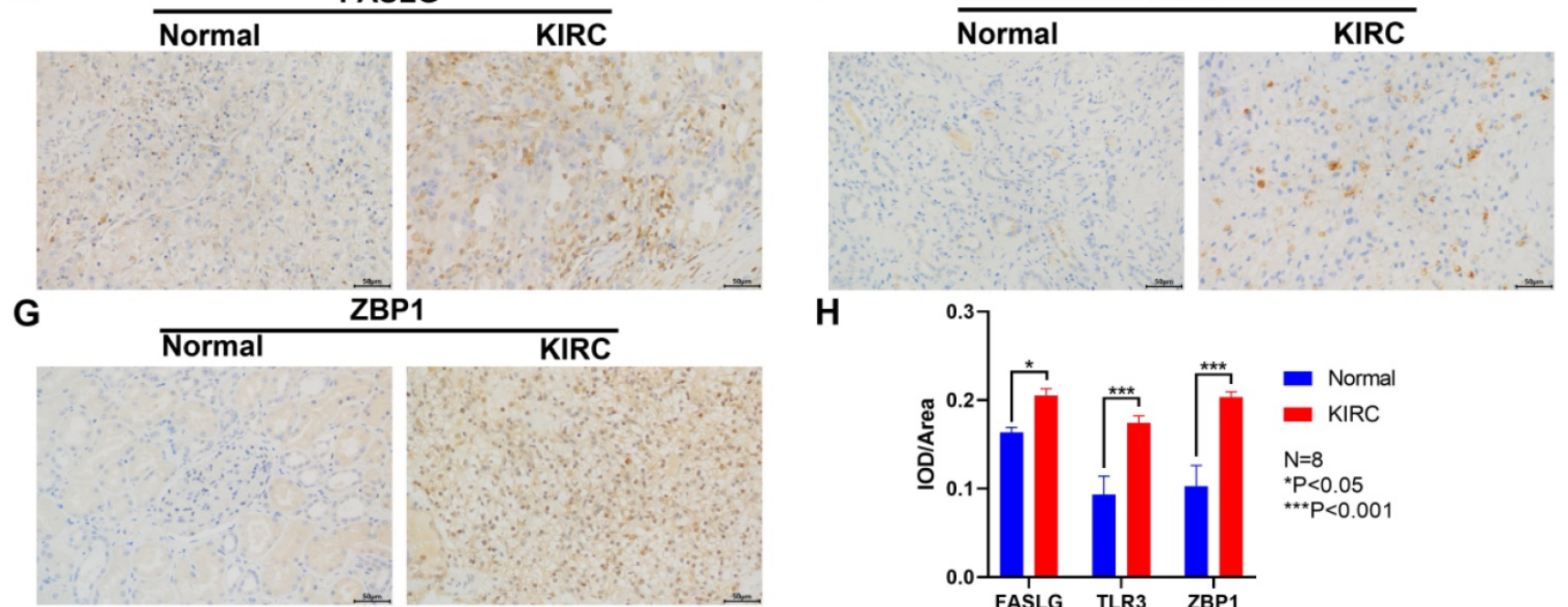

Figure 8. Differential expressions of NRGs by GEO and IHC staining validation. The comparison of FASLG, TLR3, and ZBPI expressions between KIRC and normal samples in train cohort (A) and test cohort (B). Differential expressions of FASLG, TLR3, and ZBPI validated by GSE53757 (C) and GSE40435 (D) datasets. (E-G) IHC staining was applied to validate the differential expressions of FASLG, TLR3, and ZBPI using KIRC samples from our clinical center. Scale bar $=50 \mu \mathrm{m}$. (H) Image Pro Plus 6.0 image software was applied to assess the relative expressions of FASLG, TLR3, and ZBPI which were presented as average optical density. Data are expressed as mean \pm SEM. 
A

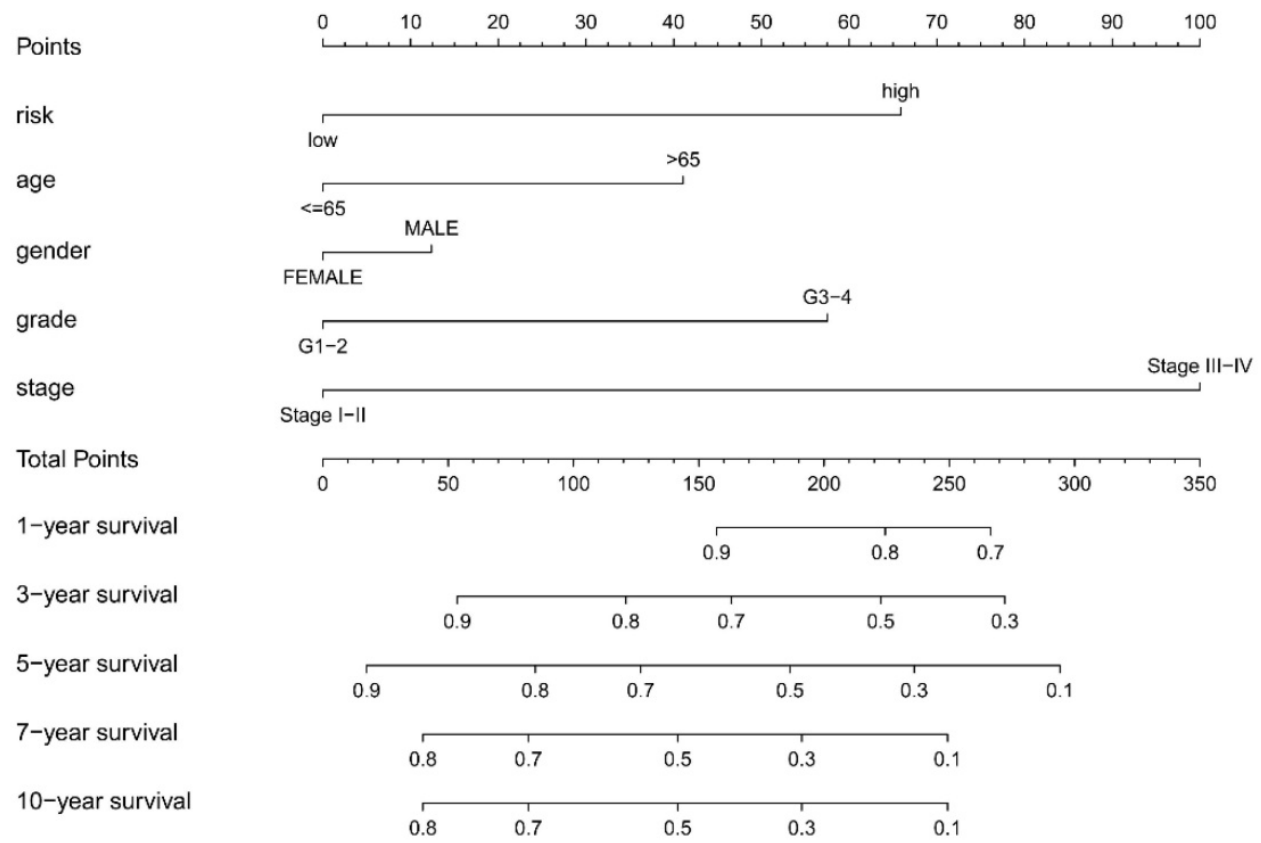

B
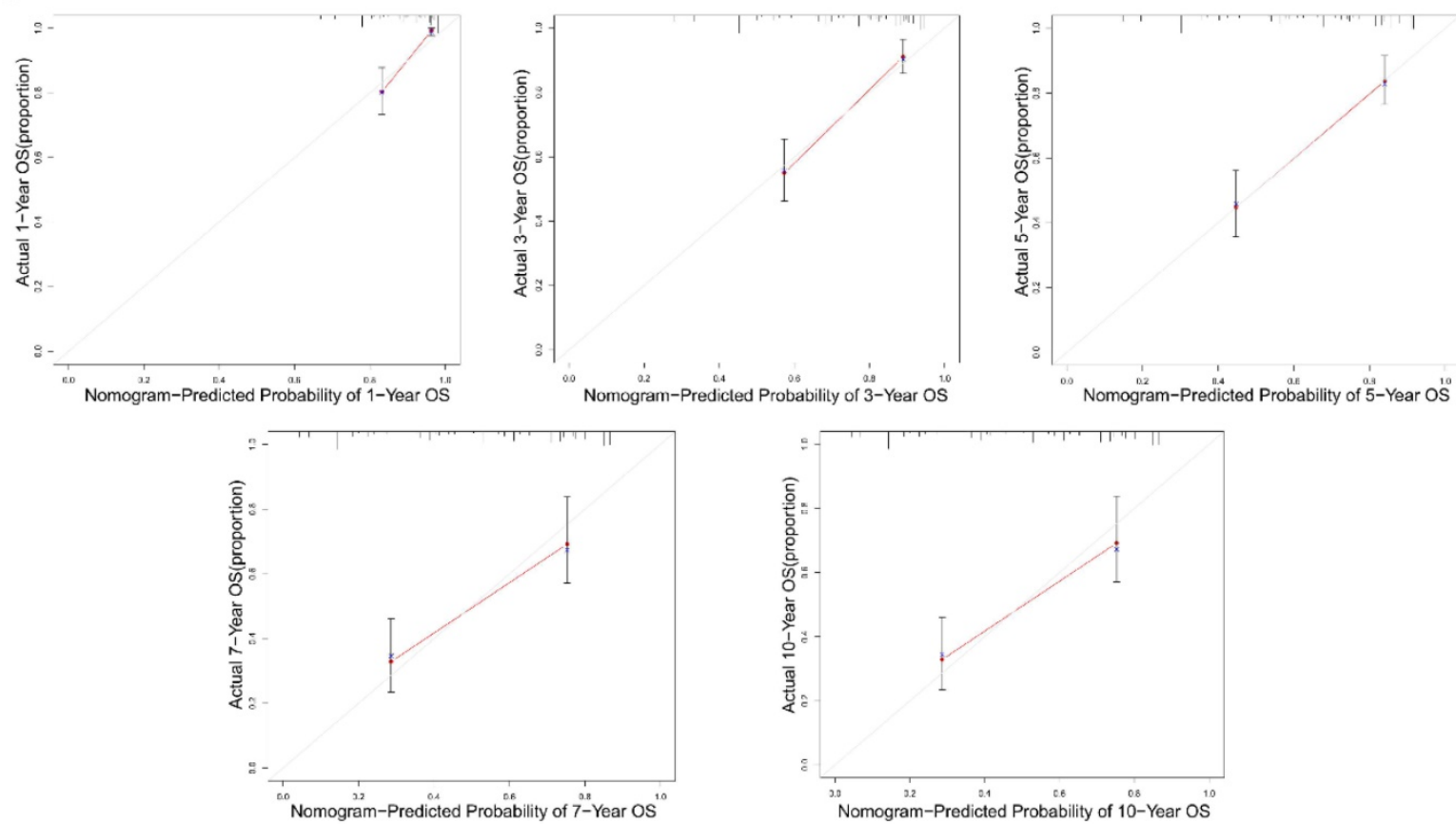

Figure 9. The construction of a nomogram for predicting survival. (A) A nomogram including risk score and clinicopathological features was constructed to predict 1/3/5/7/10-year OS. (B) The calibration plots for predicting 1/3/5/7/10-year OS based on the NRGs nomogram.

We firstly screened out the differentially expressed NRGs with prognostic values in KIRC, from which we constructed the novel risk signature consisting of three NRGs (FASLG, TLR3, and ZBP1). KIRC patients in high-risk group exhibited the upregulated expressions of FASLG and ZBP1 but a downregulated TLR3 expression. The pathway of Fas and Fas Ligand (FasL, FASL, FASLG) can initiate necroptosis upstream of RIPK3 and MLKL in renal tubular epithelial cells [34]. The cell surface FasL induces Fas-mediated killing, while autocrine secretion of soluble FasL can protect RCC cells from cytotoxic lymphocytes killing [35]. Previous studies indicated that FasL overexpression contributes to immune escape and associates with a poor prognosis in RCC $[36,37]$. As the toll-like receptor (TLR) family member, TLR3 has attracted most attention in its immune functional role, and prior studies report its high expression in immune and epithelial cells. In recent years, TLR3 overexpression is also found in multiple cancer types, but increasing evidence reveals that in tumorigenesis and progression, the dual or 
contradictory roles of TLR3 correlate with heterogeneous tumor cells and complex microenvironment [38]. TLR3/TICAM-1 axis induces necroptosis via RIPK3 and MLKL [39]. Damageassociated molecular patterns (DAMPs) released from necrotic cells, such as double-stranded RNA (dsRNA), can activate TLR3 and subsequently lead to proinflammatory response. TLR3 expression in human cancers is closely related to clinical characteristics, prognosis, metastasis, and therapy resistance [38, 40]. A study revealed that TLR3 is frequently overexpressed in both primary and metastatic KIRC [41]. ZBP1 interacts with RIPK3 to mediate tumor necroptosis [42]. The role of ZBP1 remains ambiguous in tumor progression and metastasis. Recent research indicated that ZBP1 is highly increased in late stage of mouse and human tumors, and ZBP1 deletion inhibits tumor metastasis in pre-clinical cancer models [43]. The expression of ZBP1 is increased in human cancers such as ovarian cancer and colon cancer, which is also associated with poor prognosis [44, 45]. Interestingly, we also validated the significant upregulation of FASLG, TLR3, and ZBP1 expressions through TCGA, GEO datasets, and IHC staining samples from our center. The risk score calculated based on the three-NRGs signature could independently predict survival outcome in patients with KIRC.

The tumor immune microenvironment of KIRC has a high degree of immune cells infiltration with various immunomodulatory molecules [46], which may critically impact the immunotherapeutic resistance and efficacy [47]. Based on the score comparisons of immune cell types and immune-related functions, we found that high-risk group with shorter OS possessed higher scores in macrophages, CD8 positive T cells, $\mathrm{T}$ cell co-inhibition and co-stimulation, suggesting an unbalanced and dynamic immune regulation in KIRC progression. Furthermore, the increased expressions of $\mathrm{T}$ cell exhaustion markers caused by persistent antigenic stimulation can lead to the functional loss of CD8 positive T cells [48]. Consistently, our study indicated the elevated expressions of immune checkpoint genes, including CTLA-4, LAG-3, PD-1, and SIGLEC15. The enriched analysis also revealed the critical role of immunomodulatory functions in KIRC progression, such as abnormal proportion of CD8 positive T cells, $\mathrm{T}$ helper 1 type immune response. Consistent with previous studies [49,50], our study found that cellular immune response was active in KIRC of the high-risk group. However, the overall effect of immunogenic cell death (ICD) may be limited by the defense mechanisms of tumor, such as PD-1 pathway. Interestingly, experimental evidence indicates the crosstalk between necroptosis and complementdependent cytotoxicity acting on cancer cells [51]. Therefore, the combination of targeting necroptosisrelated ICD and immunotherapy could be a novel therapeutic direction for KIRC.

Based on the TCGA dataset analyses, a novel cluster named mixed subgroup was identified and exhibited striking overexpression of mitochondrial DNA (mtDNA) [52]. Mixed subgroup affiliation was associated with a highly significant shorter OS in KIRC [52]. mtDNA has been reported to release to the cytosol of cancer cells that bear necroptosis and ZBP1 senses the cytosolic mtDNA for the initiation of cancer necroptosis under glucose deprivation or stress condition [53-55]. Furthermore, endogenous oxidatively damaged mtDNA can induce proinflammation in epithelial cells through binding to ZBP1 [56]. In our model, ZBP1 has the highest hazard ratio among three NRGs. Based on the evidence of necroptosis and ZBP1 expression, we deduce a close affiliation between the mixed subgroup and the high-risk group in our NRGs risk model. In addition, we analyzed the expression of mitochondrial and angiogenesis-related genes in our NRGs risk model. The high-risk group displayed significantly higher levels of mitochondrial gene expressions and lower levels of angiogenesis gene expressions compared to the low-risk group (Figure S3, S4). This evidence strengthens the role of the high-risk group in our model, for the upregulation of mitochondrial genes is associated with tumor growth and cancer multidrug resistance [57]. On the other hand, lower angiogenesis gene expression is associated with a poor prognosis in advanced RCC [58].

To promote the clinical transformation of the NRGs signature, we further identified the sensitive FDA-approved drugs for multiple cancer cell types with higher expressions of FASLG, TLR3, and ZBP1. Increased expression of FASLG is associated with sensitivity of cancer cells to oxaliplatin, ribociclib (LEE-011) and palbociclib. Oxaliplatin plus gemcitabine is a combination-therapeutic strategy for advanced KIRC [59], while the combination of oxaliplatin and decitabine is a promising option to treat KIRC [60]. Ribociclib and palbociclib are orally administered small-molecule inhibitors of CDK4/6, which function in synergy with other drugs to suppress KIRC in preclinical models [61-63]. The upregulated expression of TLR3 was associated with sensitivity of cancer cells to erdafitinib (JNJ-42756493) and duvelisib (IPI-45), but it was associated with increased drug resistance to tyrothricin. Erdafitinib shows preliminary clinical activity in advanced solid tumors with genomic alterations of Fibroblast Growth Factor Receptor (FGFR) pathway, which is also a 
crucial target for KIRC treatment [64, 65]. Moreover, tyrothricin complex contains gramicidin $\mathrm{A}$ as a potential agent for KIRC therapy [66, 67]. PI3K/AKT and VHL/HIF pathways are closely connected to form a large signaling network contributing to KIRC [68]. In clinical practice, a PI3K inhibitor, not duvelisib, shows anti-cancer effects on RCC [69]. The upregulation of ZBP1 expression was correlated with sensitivity of cancer cells to ceritinib (LDK-378), alectinib, and brigatinib. Recently, clinical case studies demonstrated that these small molecule inhibitors of anaplastic lymphoma kinase are promising agents for RCC precise treatment $[70,71]$.

Finally, a NRGs nomogram was constructed to predict $1 / 3 / 5 / 7 / 10$-year OS by combining NRGs risk score and clinicopathological features, showing a favorable agreement between the actual and predicted probability. However, this study still exists some limitations. Firstly, our study is retrospective in nature, requiring the use of prospective studies to validate the findings. Secondly, larger KIRC cohorts are still required to test the applicability of the necroptosis related signature. Thirdly, the novel molecular mechanisms of NRGs in KIRC need to be investigated via in vivo and in vitro experiments.

\section{Conclusions}

Based on the systematic analyses, we identified and verified a three-NRGs (TLR3, FASLG, ZBP1) risk signature with good performance in predicting survival outcome, immune microenvironment, and therapeutic sensitivity in KIRC. These findings offer molecular-level evidence to the critical role of necroptotic process in regulating immune microenvironment and therapeutic response in KIRC.

\section{Supplementary Material}

Supplementary figures.

https://www.medsci.org/v19p0377s1.pdf

\section{Acknowledgements}

This research was supported by Science and Technology Project of Wenzhou Science and Technology Bureau (Y20170390) and the Ministry of Education, Culture, Sports, Science and Technology of Japan (grant No. 21K09371). We acknowledge TCGA and GEO platform for their meaningful datasets.

\section{Ethics approval and consent to participate}

The study was conducted with the approval by Human Research Ethics Committee in the First Affiliated Hospital of Wenzhou Medical University (No. KY2021-R118), and with the informed consent from all patients.

\section{Availability of data and materials}

The datasets and primary data in this study are available from the corresponding author on reasonable request.

\section{Competing Interests}

The authors have declared that no competing interest exists.

\section{References}

1. Siegel RL, Miller KD, Fuchs HE, Jemal A. Cancer Statistics, 2021. CA Cancer J Clin. 2021; 71: 7-33.

2. Miller KD, Fidler-Benaoudia M, Keegan TH, Hipp HS, Jemal A, Siegel RL. Cancer statistics for adolescents and young adults, 2020. CA Cancer J Clin. 2020; 70: 443-59.

3. Sung H, Ferlay J, Siegel RL, Laversanne M, Soerjomataram I, Jemal A, et al. Global Cancer Statistics 2020: GLOBOCAN Estimates of Incidence and Mortality Worldwide for 36 Cancers in 185 Countries. CA Cancer J Clin. 2021; 71: 209-49.

4. Argentiero A, Solimando AG, Krebs M, Leone P, Susca N, Brunetti O, et al. Anti-angiogenesis and Immunotherapy: Novel Paradigms to Envision Tailored Approaches in Renal Cell-Carcinoma. J Clin Med. 2020; 9: 1594.

5. Lin PH, Huang CY, Yu KJ, Kan HC, Liu CY, Chuang CK, et al. Genomic characterization of clear cell renal cell carcinoma using targeted gene sequencing. Oncol Lett. 2021; 21: 169.

6. Kotecha RR, Motzer RJ, Voss MH. Towards individualized therapy for metastatic renal cell carcinoma. Nat Rev Clin Oncol. 2019; 16: 621-33.

7. Sprooten J, De Wijngaert P, Vanmeerbeerk I, Martin S, Vangheluwe P, Schlenner S, et al. Necroptosis in Immuno-Oncology and Cancer Immunotherapy. Cells. 2020; 9: 1823.

8. Gong Y, Fan Z, Luo G, Yang C, Huang Q, Fan K, et al. The role of necroptosis in cancer biology and therapy. Mol Cancer. 2019; 18: 100.

9. Tan Y, Sementino E, Cheung M, Peri S, Menges CW, Kukuyan AM, et al. Somatic Epigenetic Silencing of RIPK3 Inactivates Necroptosis and Contributes to Chemoresistance in Malignant Mesothelioma. Clin Cancer Res. 2021; 27: 1200-13.

10. Wang KJ, Wang KY, Zhang HZ, Meng XY, Chen JF, Wang P, et al. Up-Regulation of RIP3 Alleviates Prostate Cancer Progression by Activation of RIP3/MLKL Signaling Pathway and Induction of Necroptosis. Front Oncol. 2020; 10: 1720.

11. Wang Q, Wang P, Zhang L, Tessema M, Bai L, Xu X, et al. Epigenetic Regulation of RIP3 Suppresses Necroptosis and Increases Resistance to Chemotherapy in NonSmall Cell Lung Cancer. Transl Oncol. 2020; 13: 372-82.

12. Ando $\mathrm{Y}$, Ohuchida K, Otsubo $\mathrm{Y}$, Kibe S, Takesue S, Abe T, et al. Necroptosis in pancreatic cancer promotes cancer cell migration and invasion by release of CXCL5. PLoS One. 2020; 15: e0228015.

13. Yamauchi T, Fujishima F, Hashimoto M, Tsunokake J, Akaishi R, Gokon Y, et al. Necroptosis in Esophageal Squamous Cell Carcinoma: An Independent Prognostic Factor and Its Correlation with Tumor-Infiltrating Lymphocytes. Cancers (Basel). 2021; 13: 4473.

14. Chen X, Cheng B, Dai D, Wu Y, Feng Z, Tong C, et al. Heparanase induces necroptosis of microvascular endothelial cells to promote the metastasis of hepatocellular carcinoma. Cell Death Discov. 2021; 7: 33.

15. Strilic B, Yang L, Albarran-Juarez J, Wachsmuth L, Han K, Muller UC, et al. Tumour-cell-induced endothelial cell necroptosis via death receptor 6 promotes metastasis. Nature. 2016; 536: 215-8.

16. Liu ZG, Jiao D. Necroptosis, tumor necrosis and tumorigenesis. Cell Stress. 2019; 4: 1-8.

17. Snyder AG, Hubbard NW, Messmer MN, Kofman SB, Hagan CE, Orozco $\mathrm{SL}$, et al. Intratumoral activation of the necroptotic pathway components RIPK1 and RIPK3 potentiates antitumor immunity. Sci Immunol. 2019; 4: eaaw2004.

18. Van Hoecke L, Van Lint S, Roose K, Van Parys A, Vandenabeele P, Grooten J, et al. Treatment with mRNA coding for the necroptosis mediator MLKL induces antitumor immunity directed against neo-epitopes. Nat Commun. 2018; 9: 3417.

19. Ertao Z, Jianhui C, Kang W, Zhijun Y, Hui W, Chuangqi C, et al. Prognostic value of mixed lineage kinase domain-like protein expression in the survival of patients with gastric caner. Tumour Biol. 2016; 37: 13679-85.

20. He L, Peng K, Liu Y, Xiong J, Zhu FF. Low expression of mixed lineage kinase domain-like protein is associated with poor prognosis in ovarian cancer patients. Onco Targets Ther. 2013; 6: 1539-43. 
21. Li X, Guo J, Ding AP, Qi WW, Zhang PH, Lv J, et al. Association of Mixed Lineage Kinase Domain-Like Protein Expression With Prognosis in Patients With Colon Cancer. Technol Cancer Res Treat. 2017; 16: 428-34.

22. Bist P, Leow SC, Phua QH, Shu S, Zhuang Q, Loh WT, et al. Annexin-1 interacts with NEMO and RIP1 to constitutively activate IKK complex and NF-kappaB: implication in breast cancer metastasis. Oncogene. 2011; 30: 3174-85.

23. Park S, Hatanpaa KJ, Xie Y, Mickey BE, Madden CJ, Raisanen JM, et al. The receptor interacting protein 1 inhibits p53 induction through NF-kappaB activation and confers a worse prognosis in glioblastoma. Cancer Res. 2009; 69: 2809-16.

24. Lin Z, Xu Q, Miao D, Yu F. An Inflammatory Response-Related Gene Signature Can Impact the Immune Status and Predict the Prognosis of Hepatocellular Carcinoma. Front Oncol. 2021; 11: 644416.

25. Yoshihara K, Shahmoradgoli M, Martinez E, Vegesna R, Kim H, Torres-Garcia $\mathrm{W}$, et al. Inferring tumour purity and stromal and immune cell admixture from expression data. Nat Commun. 2013; 4: 2612.

26. Al-Lamki RS, Lu W, Manalo P, Wang J, Warren AY, Tolkovsky AM, et al. Tubular epithelial cells in renal clear cell carcinoma express high RIPK1/3 and show increased susceptibility to TNF receptor 1-induced necroptosis. Cell Death Dis. 2016; 7: e2287.

27. Jiao D, Cai Z, Choksi S, Ma D, Choe M, Kwon HJ, et al. Necroptosis of tumor cells leads to tumor necrosis and promotes tumor metastasis. Cell Res. 2018; 28: 868-70.

28. Li J, Huang S, Zeng L, Li K, Yang L, Gao S, et al. Necroptosis in head and neck squamous cell carcinoma: characterization of clinicopathological relevance and in vitro cell model. Cell Death Dis. 2020; 11: 391.

29. Sengupta S, Lohse CM, Leibovich BC, Frank I, Thompson RH, Webster WS, et al. Histologic coagulative tumor necrosis as a prognostic indicator of renal cell carcinoma aggressiveness. Cancer. 2005; 104: 511-20.

30. Klatte T, Chung J, Leppert JT, Lam JS, Pantuck AJ, Figlin RA, et al. Prognostic relevance of capsular involvement and collecting system invasion in stage I and II renal cell carcinoma. BJU Int. 2007; 99: 821-4

31. Vuong L, Kotecha RR, Voss MH, Hakimi AA. Tumor Microenvironment Dynamics in Clear-Cell Renal Cell Carcinoma. Cancer Discov. 2019; 9: 1349-57.

32. Aaes TL, Kaczmarek A, Delvaeye T, De Craene B, De Koker S, Heyndrickx L, et al. Vaccination with Necroptotic Cancer Cells Induces Efficient Anti-tumor Immunity. Cell Rep. 2016; 15: 274-87.

33. Yatim N, Jusforgues-Saklani H, Orozco S, Schulz O, Barreira da Silva R, Reis e Sousa C, et al. RIPK1 and NF-kappaB signaling in dying cells determines cross-priming of CD8(+) T cells. Science. 2015; 350: 328-34.

34. Zhu Y, Cui H, Lv J, Li G, Li X, Ye F, et al. Angiotensin II triggers RIPK3-MLKL-mediated necroptosis by activating the Fas/FasL signaling pathway in renal tubular cells. PLoS One. 2020; 15: e0228385.

35. Hallermalm K, De Geer A, Kiessling R, Levitsky V, Levitskaya J. Autocrine secretion of Fas ligand shields tumor cells from Fas-mediated killing by cytotoxic lymphocytes. Cancer Res. 2004; 64: 6775-82.

36. Sejima T, Isoyama T, Miyagawa I. Alteration of apoptotic regulatory molecules expression during carcinogenesis and tumor progression of renal cell carcinoma. Int J Urol. 2003; 10: 476-84.

37. Sejima T, Morizane S, Hinata N, Yao A, Isoyama T, Saito M, et al. Fas expression in renal cell carcinoma accurately predicts patient survival after radical nephrectomy. Urol Int. 2012; 88: 263-70.

38. Muresan XM, Bouchal J, Culig Z, Soucek K. Toll-Like Receptor 3 in Solid Cancer and Therapy Resistance. Cancers (Basel). 2020; 12: 3227.

39. Seya $T$, Shime $H$, Takaki $H$, Azuma M, Oshiumi $H$, Matsumoto M. TLR3/TICAM-1 signaling in tumor cell RIP3-dependent necroptosis. Oncoimmunology. 2012; 1: 917-23.

40. Zheng X, Li S, Yang H. Roles of Toll-Like Receptor 3 in Human Tumors. Front Immunol. 2021; 12: 667454

41. Morikawa T, Sugiyama A, Kume H, Ota S, Kashima T, Tomita K, et al. Identification of Toll-like receptor 3 as a potential therapeutic target in clear cell renal cell carcinoma. Clin Cancer Res. 2007; 13: 5703-9.

42. Ingram JP, Thapa RJ, Fisher A, Tummers B, Zhang T, Yin C, et al. ZBP1/DAI Drives RIPK3-Mediated Cell Death Induced by IFNs in the Absence of RIPK1. J Immunol. 2019; 203: 1348-55.

43. Baik JY, Liu Z, Jiao D, Kwon HJ, Yan J, Kadigamuwa C, et al. ZBP1 not RIPK1 mediates tumor necroptosis in breast cancer. Nat Commun. 2021; 12: 2666.

44. Dimitriadis E, Trangas T, Milatos S, Foukas PG, Gioulbasanis I, Courtis $\mathrm{N}$, et al. Expression of oncofetal RNA-binding protein CRD-BP/IMP1 predicts clinical outcome in colon cancer. Int J Cancer. 2007; 121: 486-94.

45. Gu L, Shigemasa K, Ohama K. Increased expression of IGF II mRNA-binding protein $1 \mathrm{mRNA}$ is associated with an advanced clinical stage and poor prognosis in patients with ovarian cancer. Int J Oncol. 2004; 24: 671-8.

46. Diaz-Montero CM, Rini BI, Finke JH. The immunology of renal cell carcinoma. Nat Rev Nephrol. 2020; 16: 721-35.
47. Nakano $\mathrm{O}$, Sato $\mathrm{M}$, Naito $\mathrm{Y}$, Suzuki $\mathrm{K}$, Orikasa $\mathrm{S}$, Aizawa $\mathrm{M}$, et al. Proliferative activity of intratumoral CD8(+) T-lymphocytes as a prognostic factor in human renal cell carcinoma: clinicopathologic demonstration of antitumor immunity. Cancer Res. 2001; 61: 5132-6.

48. Jiang W, He Y, He W, Wu G, Zhou X, Sheng Q, et al. Exhausted CD8+T Cells in the Tumor Immune Microenvironment: New Pathways to Therapy. Front Immunol. 2020; 11: 622509.

49. Bai D, Feng H, Yang J, Yin A, Qian A, Sugiyama H. Landscape of immune cell infiltration in clear cell renal cell carcinoma to aid immunotherapy. Cancer Sci. 2021; 112: 2126-39.

50. Senbabaoglu Y, Gejman RS, Winer AG, Liu M, Van Allen EM, de Velasco $\mathrm{G}$, et al. Tumor immune microenvironment characterization in clear cell renal cell carcinoma identifies prognostic and immunotherapeutically relevant messenger RNA signatures. Genome Biol. 2016; 17: 231.

51. Lusthaus M, Mazkereth N, Donin N, Fishelson Z. Receptor-Interacting Protein Kinases 1 and 3, and Mixed Lineage Kinase Domain-Like Protein Are Activated by Sublytic Complement and Participate in Complement-Dependent Cytotoxicity. Front Immunol. 2018; 9: 306

52. Marquardt A, Solimando AG, Kerscher A, Bittrich M, Kalogirou C, Kubler $\mathrm{H}$, et al. Subgroup-Independent Mapping of Renal Cell Carcinoma-Machine Learning Reveals Prognostic Mitochondrial Gene Signature Beyond Histopathologic Boundaries. Front Oncol. 2021; 11: 621278.

53. Chen D, Tong J, Yang L, Wei L, Stolz DB, Yu J, et al. PUMA amplifies necroptosis signaling by activating cytosolic DNA sensors. Proc Natl Acad Sci U S A. 2018; 115: 3930-5.

54. Maeda A, Fadeel B. Mitochondria released by cells undergoing TNF-alpha-induced necroptosis act as danger signals. Cell Death Dis. 2014; 5: e1312.

55. Marshall KD, Baines CP. Necroptosis: is there a role for mitochondria? Front Physiol. 2014; 5: 323.

56. Szczesny B, Marcatti M, Ahmad A, Montalbano M, Brunyanszki A, Bibli SI, et al. Mitochondrial DNA damage and subsequent activation of Z-DNA binding protein 1 links oxidative stress to inflammation in epithelial cells. Sci Rep. 2018; 8: 914

57. Yu HJ, Xiao GL, Zhao YY, Wang XX, Lan R. Targeting Mitochondrial Metabolism and RNA Polymerase POLRMT to Overcome Multidrug Resistance in Cancer. Front Chem. 2021; 9: 775226.

58. Rebuzzi SE, Perrone F, Bersanelli M, Bregni G, Milella M, Buti S. Prognostic and predictive molecular biomarkers in metastatic renal cell carcinoma patients treated with immune checkpoint inhibitors: a systematic review. Expert Rev Mol Diagn. 2020; 20: 169-85.

59. Porta C, Zimatore M, Imarisio I, Natalizi A, Sartore-Bianchi A, Danova $\mathrm{M}$, et al. Gemcitabine and oxaliplatin in the treatment of patients with immunotherapy-resistant advanced renal cell carcinoma: final results of a single-institution Phase II study. Cancer. 2004; 100: 2132-8.

60. Liu Y, Zheng X, Yu Q, Wang H, Tan F, Zhu Q, et al. Epigenetic activation of the drug transporter OCT2 sensitizes renal cell carcinoma to oxaliplatin. Sci Transl Med. 2016; 8: 348ra97.

61. Chen D, Sun X, Zhang X, Cao J. Inhibition of the CDK4/6-Cyclin D-Rb Pathway by Ribociclib Augments Chemotherapy and Immunotherapy in Renal Cell Carcinoma. Biomed Res Int. 2020; 2020: 9525207.

62. Chen T, Liu L, Zou Y, Hu X, Zhang W, Zhou T, et al. Nobiletin downregulates the SKP2-p21/p27-CDK2 axis to inhibit tumor progression and shows synergistic effects with palbociclib on renal cell carcinoma. Cancer Biol Med. 2021; 18: 227-44.

63. Wang Y, Chen S, Sun S, Liu G, Chen L, Xia Y, et al. Wogonin Induces Apoptosis and Reverses Sunitinib Resistance of Renal Cell Carcinoma Cells via Inhibiting CDK4-RB Pathway. Front Pharmacol. 2020; 11: 1152.

64. Bahleda R, Italiano A, Hierro C, Mita A, Cervantes A, Chan N, et al. Multicenter Phase I Study of Erdafitinib (JNJ-42756493), Oral Pan-Fibroblast Growth Factor Receptor Inhibitor, in Patients with Advanced or Refractory Solid Tumors. Clin Cancer Res. 2019; 25: 4888-97.

65. Sonpavde G, Willey CD, Sudarshan S. Fibroblast growth factor receptors as therapeutic targets in clear-cell renal cell carcinoma. Expert Opin Investig Drugs. 2014; 23: 305-15.

66. David JM, Owens TA, Barwe SP, Rajasekaran AK. Gramicidin A induces metabolic dysfunction and energy depletion leading to cell death in renal cell carcinoma cells. Mol Cancer Ther. 2013; 12: 2296-307.

67. Vosloo JA, Rautenbach M. Following tyrothricin peptide production by Brevibacillus parabrevis with electrospray mass spectrometry. Biochimie. 2020; 179: 101-12.

68. Guo H, German P, Bai S, Barnes S, Guo W, Qi X, et al. The PI3K/AKT Pathway and Renal Cell Carcinoma. J Genet Genomics. 2015; 42: 343-53.

69. Curigliano G, Martin M, Jhaveri K, Beck JT, Tortora G, Fazio N, et al. Alpelisib in combination with everolimus +/- exemestane in solid tumours: Phase $\mathrm{Ib}$ randomised, open-label, multicentre study. Eur J Cancer. 2021; 151: 49-62. 
70. Ross JS, Ali SM, Fasan O, Block J, Pal S, Elvin JA, et al. ALK Fusions in a Wide Variety of Tumor Types Respond to Anti-ALK Targeted Therapy. Oncologist. 2017; 22: 1444-50.

71. Varchetta V, Campanella C, Rossi M, Verzaro R, Vitale M, Soda G, et al. Identification of anaplastic lymphoma kinase fusion in clear cell renal carcinoma (ALK-tRCC): a precision oncology medicine case report. Recenti Prog Med. 2021; 112: 14e-5e. 